\title{
Existence and Uniqueness of a Regular Solution of the Cauchy-Dirichlet Problem for Doubly Nonlinear Parabolic Equations
}

\author{
A. V. Ivanov
}

Abstract. Existence and uniqueness of some Hölder continuous generalized solution of CauchyDirichlet problem for a class of degenerate or singular quasilinear parabolic equations is established. Similar equations arise in the study of turbulent filtration of a gas or a fluid through porous media.

Keywords: Quasilinear parabolic equations, generalized solutions, existence, uniqueness, Hölder estimates

AMS subject classification: $35 \mathrm{~K} 55,35 \mathrm{~K} 65,76$ A 05

\section{Introduction}

Let $\Omega$ be a bounded open set in $\mathbb{R}^{n}(n \geq 1), Q_{T}=\Omega \times(0, T], S_{T}=\partial \Omega \times(0, T]$, $\Gamma_{T}=S_{T} \cup(\bar{\Omega} \times\{t=0\})$ the parabolic boundary of the cylinder $Q_{T}$. Consider in $Q_{T}$ the equation

$$
F[u]:=\frac{\partial u}{\partial t}-\operatorname{div} a(u, \nabla u)=f
$$

where $\nabla u=\left(\frac{\partial u}{\partial x_{1}}, \ldots, \frac{\partial u}{\partial x_{n}}\right), f=f(x, t)$ is a given function and $a=\left(a^{1}, \ldots, a^{n}\right)$ is a continuous function on $\mathbb{R} \times \mathbb{R}^{n}$ satisfying for all $(u, p) \in \mathbb{R} \times \mathbb{R}^{n}$ the inequalities

$$
\begin{aligned}
a(u, p) \cdot p & \geq \nu_{0}|u|^{l}|p|^{m}-\phi_{0}(u) & & \left.\left(\nu_{0}>0, \phi_{0}(u) \geq 0\right)\right) \\
|a(u, p)| & \leq \mu_{1}|u|^{l}|p|^{m-1}+\phi_{1}(u) & & \left(m>1, l \geq 0, \phi_{1}(u) \geq 0\right) .
\end{aligned}
$$

Equations (1.1), (1.2) are known as doubly nonlinear parabolic equations. Their prototype is

$$
F_{0}[u]:=\frac{\partial u}{\partial t}-\operatorname{div}\left(|u|^{l}|\nabla u|^{m-2} \nabla u\right)=0 .
$$

In this paper we consider a special case of doubly nonlinear parabolic equations. In particular we limit ourselves by consideration equations (1.1), (1.2) only for $m>1$ and $l \geq 0$ (instead of more general conditions $m>1$ and $l>1-m$.

A. V. Ivanov: Max-Planck-Institute für Mathematik, Gottfried - Claren-Str. 26, D - 53225 Bonn; permanent address: Steklov Math. Inst., Fontanka 27, 191011 St. Petersburg, Russia 
Equations (1.1), (1.2) and in particular (1.3) arise in the study of turbulent filtration of a gas or of a fluid through porous media and non-Newtonian flows (see [13]).

Existence of generalized solutions of Cauchy-Dirichlet problem for doubly nonlinear parabolic equations were established first by Raviart [17] and J.-L. Lions [15] and then by many authors. In particular Bamberger stated in [1] his results on existence and uniqueness of some non-negative generalized solution of Cauchy-Dirichlet problem for a non-homogeneous equation $F_{0}[u]=f$ (see (1.3)).

Up to recent time there were no regularity results for doubly nonlinear parabolic equations. The simple modification of the Barenblatt explicit solutions lets to show that at least in the case $l>1$ Hölderness is the best possible smoothness of generalized solutions of equation (1.3). Hence the key question of the regularity theory for doubly nonlinear parbolic equations is establishing Hölder estimates for their generalized solutions. At first such estimates were established in [4] for the case of, so-called, doubly degenerate parabolic equations, i.e. for eqautions (1.1), (1.2) in the case $m>2$ and $l>0$.

This paper is devoted to the proof of existence and uniqueness of some Hölder continuous generalized solution of Cauchy-Dirichlet problem for equations of the type (1.1), (1.2). The crucial role is played by the Hölder estimates established by the author in $[5-9]$.

Acknowledgement. This paper was written during the stay of the author at Bonn in 1994. We would like to thank the Max-Planck-Institut für Mathematik and Professor Hirzebruch for support and hospitality.

\section{Statement of the main result}

Assume that for any $u, v \in \mathbb{R}$ and $p, q \in \mathbb{R}^{n}$ we have

(G)

$|a(u, p)| \leq \mu\left(|u|^{l}|p|^{m-1}+\bar{\mu}(|u|)\right) \quad(\mu=$ const $\geq 0, m>1, l \geq 0)$

$\bar{\mu}(s) \geq 0$ being non-decreasing.

Definition 2.1. We say that a non-negative function $u$ bounded in $Q_{T}$ is a weak solution of equation (1.1), (G) with $f \in L_{1}\left(Q_{T}\right)$ if

(a) $u \in C\left([0, T] ; L_{2}(\Omega)\right), \quad \nabla u^{\sigma+1} \in L_{m}\left(Q_{T}\right) \quad\left(\sigma=\frac{l}{m-1}\right)$

(b) for any $\phi \in C^{1}\left(\bar{Q}_{T}\right)$ with $\phi=0$ on $S_{T}$ and any $t_{1}, t_{2} \in[0, T]$

$$
\left.\int_{\Omega} u \phi d x\right|_{t_{1}} ^{t_{2}}+\int_{t_{1}}^{t_{2}} \int_{\Omega}\left(-u \phi_{t}+a\left(u, u_{x}\right) \cdot \nabla \phi-f \phi\right) d x d t=0
$$

where $u_{x}=\left(u_{x_{1}}, \ldots, u_{x_{n}}\right)$ and $u_{x_{i}}(i=1, \ldots, n)$ are defined by

$$
u_{x_{i}}=\left\{\begin{array}{ll}
(1+\sigma)^{-1} u^{-\sigma} \frac{\partial u^{\sigma+1}}{\partial x_{i}} & \text { in }\left\{Q_{T}: u>0\right\} \\
0 & \text { in }\left\{Q_{T}: u=0\right\}
\end{array} .\right.
$$


Consider the Cauchy-Dirichlet problem

$$
\begin{array}{rlrl}
F[u]:=\frac{\partial u}{\partial t}-\operatorname{div} a(u, \nabla u) & =f & \text { in } Q_{T} \\
u=\Psi & \text { on } \Gamma_{T}
\end{array}
$$

where

$$
f \in L_{1}\left(Q_{T}\right) \quad \text { and } \quad 0 \leq \Psi \in W_{1}^{1}\left(Q_{T}\right) .
$$

Definition 2.2. We say that a function $u$ is a weak solution of the Cauchy-Dirichlet problem (2.3), (2.4) if it is a weak solution of equation (1.1), (G) and $u=\Psi$ on $\Gamma_{T}$.

Remark 2.1. Every weak solution of equation (1.1), (G) and every function $\Psi \in$ $W_{1}^{1}\left(Q_{T}\right)$ have trace on $\Gamma_{T}$.

Definition 2.3. Let $\inf \left(\Psi, \Gamma_{T}\right)>0$. We say that a function $u$ is a strong solution of the Cauchy-Dirichlet problem (2.3) if it is a weak solution of (2.3) and, moreover, $\inf \left(u, Q_{T}\right)>0$ (and hence $u \in W_{m}^{1,0}\left(Q_{T}\right)$ ).

Definition 2.4. Let $\Psi \in \dot{W}_{1}^{1}\left(Q_{T}\right)$. We say that a function $u$ is a quasistrong solution of the Cauchy-Dirichlet problem (2.3) if it is a weak solution of (2.3) and, moreover, there exists a sequence $\left\{u_{n}\right\}_{n \in \mathbb{N}}$ of strong solutions of problems

$$
\begin{array}{rlrl}
F\left[u_{n}\right] & =f_{n} & \text { in } \quad Q_{T} \\
u_{n}=\Psi_{n} & \text { on } \Gamma_{T}
\end{array}
$$

such that

$$
\begin{aligned}
& u_{n} \longrightarrow u \text { in } C\left([0, T] ; L_{1}(\Omega)\right) ; \quad f_{n} \in L_{1}\left(Q_{T}\right), \quad f_{n} \longrightarrow f \text { in } L_{1}\left(Q_{T}\right) \\
& \Psi_{n}=\Psi+\varepsilon_{n}(x, t) \\
& \varepsilon_{n} \in W_{1}^{1}\left(Q_{T}\right) \cap C\left(\bar{Q}_{T}\right), \quad \inf \left(\varepsilon_{n}, \Gamma_{T}\right)>0, \quad \sup \left(\varepsilon_{n}, \Gamma T\right) \rightarrow 0 .
\end{aligned}
$$

Definition 2.5. Let $\Psi \in \dot{W}_{1}^{1}\left(Q_{T}\right)$. We say that a function $u$ is a regular solution of the Cauchy-Dirichlet problem (2.3) if it is Hölder continuous in $\bar{Q}_{T}$ and a quasistrong solution of equation (2.3).

Introduce the following assumptions:

$(\Omega)\left|B_{\rho}(x) \cap \Omega\right| \leq\left(1-\alpha_{0}\right)\left|B_{\rho}(x)\right|\left(x \in \partial \Omega, \rho \in\left(0, \rho_{0}\right)\right)$ for some $\rho_{0}>0, \alpha_{0} \in(0,1)$

(BI) $0 \leq \Psi \in \dot{W}_{2}^{1}\left(Q_{T}\right) \cap C_{\beta, \beta / m}\left(\Gamma_{T}\right) \quad(\beta \in(0,1))$

(RHS) $0 \leq f \in L_{\infty}\left(Q_{T}\right)$.

Moreover, assume that the following conditions are fulfilled for equation (1.1):

0) The functions $u^{-\alpha} a^{i}\left(u, u^{-\alpha} p\right) \quad\left(i=1, \ldots, n ; \alpha=\frac{l}{m}\right)$ are continuous on $\bar{R}_{+} \times \mathbb{R}^{n}$. 
1) (Growth condition). For any $u \in \bar{R}_{+}$and $p \in \mathbb{R}^{n}$

$$
\begin{aligned}
& a(u, p) \cdot p \geq \nu_{0}|u|^{l}|p|^{m}-\mu_{0}\left(|u|^{\delta}+1\right) \quad\left(\nu_{0}>0 ;\left\{\begin{array}{ll}
2<\delta<m+l & \text { if } m+l>2 \\
\delta=2 & \text { if } m+l \leq 2
\end{array}\right)\right. \\
& |a(u, p)| \leq \mu_{1}|u|^{\prime}|p|^{m-1}+\mu(|u|)|u|^{\alpha}\left(\alpha=\frac{l}{m}, \mu(s) \geq 1 \text { non-decreasing on } \mathbb{R}_{+}\right) .
\end{aligned}
$$

2) (Strict monotonicity condition). There exists a constant $\nu_{1}>0$ and a continuous vector function $b: \mathbb{R} \rightarrow \mathbb{R}^{n}$ such that for any $u \in \mathbb{R}$ and $p, q \in \mathbb{R}$

$$
(a(u, p)-a(u, q)) \cdot(p-q) \geq \nu_{1}|u|^{l}|p-q|^{\kappa}\left(|p-b|^{m}+|q-b|^{m}\right)^{1-\kappa / m}
$$

where $\kappa=m$ if $m \geq 2$ and $\kappa=2$ if $1<m<2$.

3) (Local Lipschitz condition). For any $u, v \in[\varepsilon, M](0<\varepsilon<M)$ and any $p \in \mathbb{R}^{n}$

$$
|a(u, p)-a(v, p)| \leq \Lambda|u-v|\left(1+|p|^{m-1}\right)
$$

where $\Lambda=\Lambda(\varepsilon, M) \geq 0$.

4) $(m, l) \in D \backslash \omega$ where

$$
\begin{aligned}
& D=\{(m, l): m>1, l \geq 0\} \\
& \omega=\left\{(m, l) \in D: \frac{\sigma+1}{\sigma+2} \leq \frac{1}{m}-\frac{1}{n}, \sigma=\frac{l}{m-1}\right\} .
\end{aligned}
$$

Theorem 2.1 (Existence and uniqueness of regular solution). Let conditions $(\Omega)$, (BI), (RHS) and 0) - 4) hold. Then the Cauchy-Dirichlet problem (2.3) has exactly one regular solution.

Remark 2.2. Conditions 0) - 3) are fulfilled for equation (1.3).

Remark 2.3. It is easy to see that $\Omega \subset F:=\{(m, l) \in D: m+l<2\}$. We constructed a counter-example (see [10]) showing that for every $(m, l) \in \omega$ the local boundedness of generalized solutions of equation (1.3) fails to be true.

Remark 2.4. Existence of Hölder continuous weak solution of the Cauchy-Dirichlet problem for some class of equations of the type (1.1), (1.2) in the case $m \geq 2$ and $l \geq 0$ was proved in [11]. Existence and uniqueness of regular solution of the CauchyDirichlet problem (2.3) under conditions ( $\Omega$ ), (BI), (RHS) and 0 ) - 3) and for $l \geq 0$, $\max \left(1, \frac{2 n}{n+2}\right)<m<2, m+l>2$ can be derived from results of [12]. The proofs of the results of [11] and [12] are based on using Hölder estimates established in [4] and [5 9], respectively. 


\section{Uniqueness of quasistrong solution}

In this section we state the uniqueness results of paper [12]. Assume at first that for any $u, v \in \mathbb{R}$ and any $p, q \in \mathbb{R}^{n}$ the function $a=\left(a^{1}, \ldots, a^{n}\right)$ satisfies the following conditions:

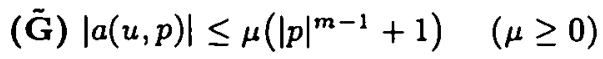

(M) $(a(u, p)-a(u, q)) \cdot(p-q) \geq 0$

(L) $|a(u, p)-a(v, p)| \leq \lambda|u-v|\left(|p|^{m-1}+1\right) \quad(\Lambda=$ const $\geq 0, m>1)$.

Definition 3.1. We say that a function $u$ is a generalized solution of equation (1.1), $(\tilde{\mathrm{G}})$ if $u \in W_{m}^{1,0}\left(Q_{T}\right) \cap C\left([0, T] ; L_{1}(\Omega)\right)$ and for all $0 \leq \phi \in W_{m}^{1}\left(Q_{T}\right) \cap L_{\infty}\left(Q_{T}\right)$ and any $t_{1}, t_{2} \in[0, T]$

$$
\left.\int_{\Omega} u \phi d x\right|_{t_{1}} ^{t_{2}}+\int_{\Omega}\left(-u \phi_{t}+a(u, \nabla u) \cdot \nabla \phi-f \phi\right) d x d t=0
$$

Analogously, the function $u$ is called subsolution and supersolution if in (3.1) the sign $"="$ is replacesd by " $\leq "$ and " $\geq "$, respectively.

Proposition 3.1 (Comparison Principle, see [12]). Assume that conditions ( $\tilde{\mathrm{G}})$, (M) and (L) hold. Let $u_{1}$ and $u_{2}$ be a generalized subsolution and a supersolution, repsectively, such that

$$
F\left[u_{1}\right] \leq f_{1} \quad \text { and } \quad F\left[u_{2}\right] \geq f_{2}
$$

where $f_{1}, f_{2} \in L_{1}\left(Q_{T}\right)$. If $u_{1} \leq u_{2}$ on $S_{T}=\partial \Omega \times(0, T]$, then for any $\tau \in(0, T]$ we have

$$
\begin{aligned}
\left.\int_{\Omega}\left(u_{1}-u_{2}\right)^{+} d x\right|^{t=\tau} \leq & \left.\int_{\Omega}\left(u_{1}-u_{2}\right)^{+} d x\right|^{t=0} \\
& +\int_{0}^{\tau} \int_{\Omega}\left(f_{1}-f_{2}\right) \operatorname{sign}\left(u_{1}-u_{2}\right)^{+} d x d t .
\end{aligned}
$$

Proof. Let $0 \leq \eta \in \stackrel{\circ}{W}_{m}^{1,0}\left(Q_{T}\right) \cap L_{\infty}\left(Q_{T}\right), 0<h<t_{0}<t_{2}<T-h, Q_{t_{1, t_{2}}}:=\Omega \times$ $\left[t_{1}, t_{2}\right]$. Then from the conditions of Proposition 3.1 it follows (see also [14:pp.167, 477]) that

$$
\begin{aligned}
\iint_{Q_{1_{1}, t_{2}}}\left\{\left(u_{1}-u_{2}\right)_{\bar{h} \iota} \eta+\left(\left(a\left(u_{1}, \nabla u_{1}\right)\right)_{\bar{h}}\right.\right. & \left.\left.-\left(a\left(u_{2}, \nabla u_{2}\right)\right)_{\bar{h}}\right) \cdot \nabla \eta\right\} d x d t \\
& \leq \iint_{Q_{t_{1}, \ell_{2}}}\left(f_{1}-f_{2}\right)_{\bar{h}} \eta d x d t
\end{aligned}
$$

where

$$
g_{\bar{h}}=\frac{1}{h} \int_{t-h}^{t} g(x, \tau) d \tau
$$


Denote

$$
H_{\delta}(s)=\left\{\begin{array}{ll}
1 & \text { if } s \geq \delta \\
\frac{s}{\delta} & \text { if } 0<s<\delta \\
0 & \text { if } s \leq 0
\end{array} \quad \text { and } \quad G_{\delta}(s)= \begin{cases}s-\frac{\delta}{2} & \text { if } s \geq \delta \\
\frac{s^{2}}{2 \delta} & \text { if } 0<s<\delta \\
0 & \text { if } s \geq 0\end{cases}\right.
$$

so that $G_{\delta}^{\prime}(s)=H_{\delta}(s)$ on $\mathbb{R}$. Set in $(3.3)$

$$
\eta=H_{\delta}\left(u_{1}-u_{2}\right) \text {. }
$$

Obviously that the test function (3.4) is admissible. In view of the concavity of the function $G_{6}$ we have

$$
\left(u_{1}-u_{2}\right)_{\bar{h}_{t}} H_{\delta}\left(u_{1}-u_{2}\right) \geq\left(G_{\delta}\left(u_{1}-u_{2}\right)\right)_{\bar{h} t} .
$$

Then from (3.3) it follows that

$$
\begin{aligned}
& \iint_{Q_{t_{1}, i_{2}}}\left(G_{\delta}\left(u_{1}-u_{2}\right)\right)_{\bar{h} t} d x d t \\
& \quad+\iint_{Q_{t_{1}, t_{2}}}\left(\left(a\left(u_{1}, \nabla u_{1}\right)\right)_{\bar{h}}-\left(a\left(u_{2}, \nabla u_{2}\right)\right)_{\bar{h}}\right) \cdot \nabla\left(u_{1}-u_{2}\right) H_{\delta}^{\prime}\left(u_{1}-u_{2}\right) d x d t \\
& \quad \leq \iint_{Q_{t_{1}, t_{2}}}\left(f_{1}-f_{2}\right)_{\bar{h}} H_{\delta}\left(u_{1}-u_{2}\right) d x d t .
\end{aligned}
$$

Using the Newton-Leibnitz formula for the first term in (3.5)and then letting $h \rightarrow 0$ we obtain for any $\tau \in(0, T]$

$$
\begin{aligned}
& \left.\int_{\Omega} G_{\delta}\left(u_{1}-u_{2}\right) d x\right|_{0} ^{\top} \\
& \quad+\frac{1}{\delta} \iint_{\left\{Q_{0, r}: 0<u_{1}-u_{2}<\delta\right\}}\left(a\left(u_{1}, \nabla u_{1}\right)-a\left(u_{2}, \nabla u_{2}\right)\right) \cdot \nabla\left(u_{1}-u_{2}\right) d x d t \\
& \quad \leq \iint_{Q_{0, r}}\left(f_{1}-f_{2}\right) H_{\delta}\left(u_{1}-u_{2}\right) d x d t .
\end{aligned}
$$

Taking into account that

$$
G_{\delta}\left(u_{1}-u_{2}\right) \longrightarrow\left(u_{1}-u_{2}\right)^{+} \quad \text { and } \quad H_{\delta}\left(u_{1}-u_{2}\right) \longrightarrow \operatorname{sign}\left(u_{1}-u_{2}\right)^{+}
$$

as $\delta \rightarrow 0$ we derive from (3.6) and conditions $(M)$ and $(L)$ that inequality (3.2) holds

Consider now the Cauchy-Dirichlet problem (2.3) assuming that condition $(\tilde{G})$ holds and $f \in L_{1}\left(Q_{T}\right), \Psi \in W_{1}^{1}\left(Q_{T}\right)$.

Definition 3.2. We say that a function $u$ is a generalized solution of the CauchyDirichlet problem (2.3) if it is a generalized solution of equation (1.1) and $u=\Psi$ on $\Gamma_{T}$.

From Proposition 3.1 we can derive directly the following 
Proposition 3.2. Let conditions $(\tilde{\mathrm{G}}),(\mathrm{M})$ and $(\mathrm{L})$ are fulflled. Then there is at most one generalized solution of the Cauchy-Dirichlet problem (2.3).

Replace now condition ( $\tilde{G})$ by condition $(G)$ (see Section 2) and consider instead of assumption (L) the local Lipschitz condition

$$
\begin{gathered}
\text { (i்) }|a(u, p)-a(v, p)| \leq \Lambda|u-v|\left(1+|p|^{m-1}\right) \quad(\Lambda=\Lambda(\varepsilon, M) \geq 0) \\
\text { for any } u, v \in[\varepsilon, M] \quad(0<\varepsilon<M) \text { and any } p \in \mathbb{R}^{n} .
\end{gathered}
$$

From Proposition 3.2 we can derive the following

Proposition 3.3. Let $\inf \left(\Psi, \Gamma_{T}\right)>0$ and let conditions (G), (M) and ( $\left.\tilde{\mathrm{L}}\right)$ hold. Then there is at most one strong (in sense of Definition 2.3) solution of the CauchyDirichlet problem (2.3).

The main uniqueness result for doubly nonlinear parabolic equations is the following

Theorem 3.1 (Uniqueness of quasistrong solution, see [12]). Let $\Psi \in \dot{W}_{1}^{1}\left(Q_{T}\right)$ and

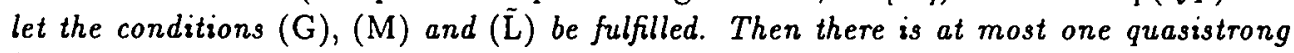
(in sense of Definition 2.4) solution of the Cauchy-Dirichlet problem (2.3).

Proof. Let $u$ and $\tilde{u}$ be two quasistrong solutions of problem (2.3). Let $\left(u_{n}, f_{n}, \Psi_{n}\right)$ $\rightarrow(u, f, \Psi)$ and $\left(\tilde{u}_{n}, \tilde{f}_{n}, \tilde{\Psi}_{n}\right) \rightarrow(\tilde{u}, f, \Psi)$ in sense of (2.5). Obviously we can choose subsequences $\left\{\Psi_{n}\right\}$ and $\left\{\tilde{\Psi}_{n}\right\}$ such that $\sup \left(\Psi_{n}, S_{T}\right) \leq \inf \left(\tilde{\Psi}_{n}, S_{T}\right) \quad(n \in \mathbb{N})$. Then we can apply Proposition 3.1, i.e., for any $\tau \in(0, T]$

$$
\left.\int_{\Omega}\left(u_{n}-\tilde{u}_{n}\right)^{+} d x\right|^{t=r} \leq \int_{\Omega}\left(\Psi_{n}-\tilde{\Psi}_{n}\right) d x+\iint_{0 \Omega}^{\tau}\left|f_{n}-\tilde{f}_{n}\right| d x d t
$$

Letting $n \rightarrow \infty$ and using (2.5) we obtain that $(u-\tilde{u})^{+}=0$ a.e. in $Q_{T}$

Remark 3.1. In some sense Definition 2.4 of quasistrong solution and Theorem 3.1 are similar to the definition of "limit of strong solutions" and the corresponding uniqueness theorem given by Bamberger [1] for equation (1.3). However instead of our condition inf $\left(u, Q_{T}\right)>0$ in the definition of strong solution Bamberger used condition $\frac{\partial u}{\partial t} \in L_{1}\left(Q_{T}\right)$.

We introduce now the following

Definition 3.3. We say that a function $u$ is a maximal weak solution of the CauchyDirichlet problem (2.3) if it is a weak solution of problem (2.3) and, moreover, for any weak solution $v$ of this problem we have

$$
u(x, t) \geq v(x, t) \quad \text { in } Q_{T} .
$$

The reason of uniqueness of quasistrong solution of equation (2.3) can be found by means the following proposition that easily follows from the proof of Proposition 3.1 given in [12]. 
Proposition 3.4 (A. V. Ivanov, W. Jäger and P. Z. Mkrtychyan). Every quasistrong solution of the Cauchy-Dirichlet problem (2.1) is a maximal weak solution of this problem.

Proof. Inequality (3.3) remains valid for any weak solutions $u_{1}$ and $u_{2}$ of the Cauchy-Dirichlet problem if we change $\nabla u_{1}$ and $\nabla u_{2}$ in (3.3) by $\left(u_{1}\right)_{x}$ and $\left(u_{2}\right)_{x}$, respectively (where $u_{x}$ is defined by (2.2)). Let $u$ be a quasistrong and $v$ be a weak solution of (2.3). Consider the sequence $\left\{u_{(n)}\right\}$ of strong solutions of problems

$$
\begin{aligned}
\mathcal{F}\left[u_{(n)}\right] & =f_{n} & & \text { in } Q_{T} \\
u_{(n)} & =\Psi_{n} & & \text { on } \Gamma_{T}
\end{aligned}
$$

satisfying conditions (2.5). In particular,

$$
u_{(n)} \geq \varepsilon_{(n)} \quad \text { and } \quad \varepsilon_{(n)}=\inf \left(\varepsilon_{n}, Q_{T}\right)>0 \quad(n \in \mathbb{N}) .
$$

Consider (3.3) in the case $u_{1}=v$ and $u_{2}=u_{(n)}$. Set in (3.3)

$$
\eta=H_{\delta}\left(v-u_{(n)}\right)
$$

where $H_{\delta}(s)$ is defined like above. Such a test function is admissible for (3.3) because

$$
\left.H_{\delta}\left(v-u_{(n)}\right)=H_{\delta}\left(\left(v-u_{(n)}\right)^{+}\right)=H_{\delta}\left(\left(v_{(n)}-u_{(n)}\right)\right)^{+}\right)
$$

where $v_{(n)}=\sup \left(v, \varepsilon_{(n)}\right)$. Therefore, function (3.8) belongs to $\mathscr{W}_{m}^{1,0}\left(Q_{T}\right) \cap L_{\infty}\left(Q_{T}\right)$. Then repeating arguments of the proof of Proposition 3.1 we obtain

$$
\left.\int_{\Omega}\left(v-u_{(n)}\right)^{+} d x\right|^{t=\tau} \leq 0 \quad \text { for any } \quad \tau \in(0, T] .
$$

Using (2.5) we derive from (3.9) that $v-u \leq 0$ a.e. in $\Omega$, i.e., inequality (3:7) is established

\section{Hölder estimates for doubly nonlinear parabolic equations}

Establishing Hölder estimates is the key question of the regularity problem for doubly nonlinear parabolic equations not only in view of the fact that Hölderness is the best possible smoothness for a large class of such equations. In fact Hölder estimates for bounded generalized solutions are crucial and the best difficult step in proving of existence of regular solution of Cauchy-Dirichlet problem for doubly nonlinear parabolic equations.

Directly from our results [5 - 9] for doubly nonlinear parabolic equations of the full type

$$
\frac{\partial u}{\partial t}-\operatorname{div} a(x, t, u, \nabla u)+a_{0}(x, t, u, \nabla u)=0
$$


with the limit growth conditions we can derive the following estimates for equations of the type (1.1), (1.2). Introduce condition

$$
\begin{aligned}
& \text { (H) } a=\left(a^{1}, \ldots, a^{n}\right) \text { is continuous on } \mathbb{R} \times \mathbb{R}^{n} \quad(i=1, \ldots, n) \\
& a(u, p) \cdot p \geq \nu_{0}|u|^{l}|p|^{m}-\varphi_{0} \quad\left(\nu_{0}>0\right) \\
& |a(u, p)| \leq \mu_{1}|u|^{\prime}|p|^{m-1}+|u|^{\alpha} \varphi_{1} \quad\left(\alpha=\frac{l}{m}\right) \\
& |f(x, t)| \leq \varphi_{2}
\end{aligned}
$$

where $\varphi_{i}=$ const $\geq 0 \quad(i=0,1,2)$. For the sake of brevity we state here only global Hölder estimates (i.e. Hölder estimates up to the boundary) for equations (1.1), (1.2).

Theorem 4.1 (see $[5-8])$. Assume that $m+l \geq 2$ and let conditions $(\mathrm{H})$ and $(\Omega)$ hold. Let $u$ be a weak solution of equation (1.1) (in sense of Definition 2.1) such that its trace on the parabolic boundary $\Gamma_{T}$ is Holder continuous. Then function u belongs to the class $C^{\lambda, \lambda / m}\left(\bar{Q}_{T}\right)$ for some $\lambda \in(0,1)$. Moreover

$$
\langle u\rangle_{\lambda, \bar{Q}_{T}}:=\sup _{(x, t),\left(x^{\prime}, t^{\prime}\right) \in Q_{T}} \frac{\left|u(x, t)-u\left(x^{\prime}, t^{\prime}\right)\right|}{\left(\left|x-x^{\prime}\right|^{m}+\left|t-t^{\prime}\right|\right)^{\lambda / m}} \leq K
$$

where $\lambda \in(0,1)$ and $K>0$ depend only on $\sup \left(u, Q_{T}\right), n, m, l, \nu_{0}, \mu_{0}, \varphi_{0}, \varphi_{1}, \varphi_{2}$, $|\Omega|, T, \alpha_{0}, \rho_{0}$ and the Hölder constant and exponent of the trace of function $u$ on $\Gamma_{T}$.

Theorem 4.2 (see [9]). Assume that $m+l<2$ and let conditions (H), (M), (L) and $(\Omega)$ hold. Let $u \in W_{m}^{1,0}\left(Q_{T}\right)$ be a weak solution of equation (1.1) (in sense of Definition 2.1) such that its trace on the parabolic boundary $\Gamma_{T}$ is Hölder continuous. Then $u$ belongs to $C^{\lambda, \lambda / m}\left(\bar{Q}_{T}\right)$ for some $\lambda \in(0,1)$. Moreover estimate (4.2) holds with some constants $\lambda \in(0,1)$ and $K>0$ depending on the same data as in the case of Theorem 4.1 (in particular $\lambda$ and $K$ are independent of $\|\nabla u\|_{L_{m}\left(Q_{T}\right)}$ and the constant $\Lambda$ from condition $(L))$.

Remark 4.1. Theorems 4.1 and 4.2 remain valid if the inequalities in condition (H) are fulfilled only for values $u$ from the range of weak solution under consideration.

Remark 4.2. The proofs of Theorems 4.1 and 4.2 (as well as Hölder estimates for general equations (4.1) in [5 - 9]) are concerned with some development of the methods of papers by De Giorgi, Ladyzhenskaya and Ural'tseva (see [14]), DiBenedetto [3], Chen and DiBenedetto [2], and the author [4].

Remark 4.3. Other results on Hölder estimates for some classes of doubly nonlinear parabolic equations are obtained in $[16,18]$. 


\section{The auxiliary Cauchy-Dirichlet problem}

This section has an auxiliary character. At first we prove some generalization of the well-known Friedrieks inequality (cf. [14: pp. 529 - 530]) which will be used not only in this section.

Lemma 5.1. Let $\left\{\Psi_{\kappa}\right\}$ be an orthonormal basis in $L_{2}(\Omega)$ and let $\beta \geq 0$ be fixed. Then for any $\varepsilon>0$ there exists a number $\mathcal{N}_{\epsilon}$ such that for any function $u$ satisfying the condition

$$
|u|^{\beta} u \in \stackrel{\circ}{W}_{m}^{1}(\Omega) \quad\left(m>1, \frac{1}{m}<\frac{1}{n}+\frac{1+\beta}{2}\right)
$$

we have

$$
\|u\|_{L_{2}(\Omega)} \leq\left(\sum_{k=1}^{\mathcal{N}_{\varepsilon}}\left(u, \Psi_{\kappa}\right)^{2}\right)^{1 / 2}+\varepsilon\left\|\nabla\left(|u|^{\beta} u\right)\right\|_{L_{m}(\Omega)}^{1 /(\beta+1)}
$$

where $\left(u, \Psi_{\kappa}\right):=\int_{\Omega} u \Psi_{\kappa} d x$ and $\mathcal{N}_{\varepsilon}$ does not depend on $u$.

Proof. It is sufficient to prove that for any $\delta>0$ and $\varepsilon>0$

$$
\|u\|_{L_{2}(\Omega)} \leq(1+\delta)\left(\sum_{k=1}^{\mathcal{N}_{\varepsilon, \delta}}\left(u, \Psi_{\kappa}\right)^{2}\right)^{1 / 2}+\varepsilon\left\|\nabla\left(|u|^{\beta} u\right)\right\|_{L_{m}(\Omega)}^{1 /(\beta+1)}
$$

Really, for the function $v=|u|^{\beta} u$ we have the well-known Sobolev inequality

$$
\|v\|_{L_{r}(\Omega)} \leq c\|\nabla v\|_{L_{m}(\Omega)} \quad\left(r=\frac{2}{1+\beta}>0\right)
$$

because from condition $\frac{1}{m}<\frac{1}{n}+\frac{1+\beta}{2}$ it follows that $\frac{1}{r}>\frac{1}{m}-\frac{1}{n}$. Rewrite (5.4) as

$$
\|u\|_{L_{2}(\Omega)} \leq c_{1}\left\|\nabla\left(|u|^{\beta} u\right)\right\|_{L_{m}(\Omega)}^{1 /(\beta+1)} .
$$

Then from (5.3) and (5.5) it follows that

$$
\|u\|_{L_{2}(\Omega)} \leq\left(\sum_{k=1}^{\mathcal{N}_{\varepsilon, \delta}}\left(u, \Psi_{\kappa}\right)^{2}\right)^{1 / 2}+\left(c_{1} \delta+\varepsilon\right)\left\|\nabla\left(|u|^{\beta} u\right)\right\|_{L_{m}(\Omega)}^{1 /(\beta+1)}
$$

i.e., the result of Lemma 5.1 is true. So prove that (5.3) holds.

If (5.3) is violated, then there exist an $\varepsilon_{0}>0$ and a sequence of functions $\left\{u_{\nu}\right\}$ satisfying condition (5.1) such that for some fixed $\delta>0$ and any $\nu \in \mathbb{N}$

$$
\left\|u_{\nu}\right\|_{L_{2}(\Omega)}>(1+\delta)\left(\sum_{k=1}^{\nu}\left(u_{\nu}, \Psi_{k}\right)^{2}\right)^{1 / 2}+\varepsilon_{0}\left\|\nabla\left(\left|u_{\nu}\right|^{\beta} u_{\nu}\right)\right\|_{L_{m}(\Omega)}^{1 /(\beta+1)}
$$


Then for functions $\hat{u}_{\nu}=u_{\nu} /\left\|u_{\nu}\right\|_{L_{2}(\Omega)}$ we have

$$
1=\left\|\hat{u}_{\nu}\right\|_{L_{2}(\Omega)}>(1+\delta)\left(\sum_{k=1}^{\nu}\left(\hat{u}_{\nu}, \Psi_{k}\right)^{2}\right)^{1 / 2}+\varepsilon_{0}\left\|\nabla\left(\left|\hat{u}_{\nu}\right|^{\beta} \hat{u}_{\nu}\right)\right\|_{L_{m}(\Omega)}^{1 /(\beta+1)}
$$

Denote $v_{\nu}=\left|\hat{u}_{\nu}\right|^{\beta} \hat{u}_{\nu}$. In view of (5.8) the norms $\left\|\nabla v_{\nu}\right\|_{L_{m}(\Omega)}$ are uniformly bounded and hence (taking into account that $\frac{1}{r}>\frac{1}{m}-\frac{1}{n}$ for $r=\frac{2}{1+\beta}$ ) there exists some subsequence $\left\{v_{\nu_{0}}\right\}$ converging strongly in $L_{r}(\Omega)$. It is easy to see then that the subsequence $\left\{u_{\nu_{1}}\right\}$ converges strongly in $L_{2}(\Omega)$ to some function $\hat{u} \in L_{2}(\Omega)$. Really, in view of the strict monotonicity of the function $x \rightarrow|x|^{\beta} x(\beta>0)$ we have

$$
c^{-1}\left|\hat{u}_{\nu}-\hat{u}_{\mu}\right|^{2+\beta} \leq\left(\left|\hat{u}_{\nu}\right|^{\beta} \hat{u}_{\nu}-\left|\hat{u}^{\mu}\right|^{\beta} \hat{u}_{\mu}\right)\left(\hat{u}_{\nu}-\hat{u}_{\mu}\right) \leq\left|v_{\nu}-v_{\mu}\right|\left|\hat{u}_{\nu}-\hat{u}_{\mu}\right|
$$

with some constant $c>0$ and hence

$$
\left|\hat{u}_{\nu}-\hat{u}_{\mu}\right|^{2} \leq c\left|v_{\nu}-v_{\mu}\right|^{r} \quad\left(r=\frac{2}{1+\beta}\right) .
$$

Moreover, it is obvious that $\|\hat{u}\|_{L_{2}(\Omega)}=1$. The functions $P_{\nu_{0}} \hat{u}_{\nu_{0}}=\sum_{k=1}^{\nu_{1}}\left(\hat{u}_{\nu_{0}}, \Psi_{k}\right) \Psi_{k}$ also converge strongly in $L_{2}(\Omega)$ to $\hat{u}$ because

$$
\begin{aligned}
\left\|\hat{u}-P_{\nu_{0}} \hat{u}_{\nu_{0}}\right\|_{L_{2}(\Omega)} & =\left\|P_{\nu_{0}}\left(\hat{u}-\hat{u}_{\nu_{0}}\right)+\left(E-P_{\nu_{0}}\right) \hat{u}\right\|_{L_{2}(\Omega)} \\
& \leq\left\|\hat{u}-\hat{u}_{\nu_{0}}\right\|_{L_{2}(\Omega)}+\left\|\left(E-P_{\nu_{s}}\right) \hat{u}\right\|_{L_{2}(\Omega)} \\
& \rightarrow 0 \quad \text { as } s \rightarrow \infty
\end{aligned}
$$

Then

$$
\left(\sum_{k=1}^{\nu_{0}}\left(\hat{u}_{\nu_{1}}, \Psi_{k}\right)^{2}\right)^{1 / 2}=\left\|P_{\nu_{0}} \hat{u}_{\nu_{1}}\right\|_{L_{2}(\Omega)} \longrightarrow\|\hat{u}\|_{L_{2}(\Omega)}=1 \quad \text { as } s \rightarrow \infty .
$$

In view of (5.8), (5.9) we obtain then the impossible inequality $1 \geq 1+\delta$

Now we consider the Cauchy-Dirichlet problem

$$
\begin{array}{rlr}
F[u]=f & \text { in } Q_{T} \\
u=\Psi & \text { on } \Gamma_{T}
\end{array}
$$

assuming the following:

$\left.\mathbf{0}^{\prime}\right) a=\left(a^{1}, \ldots, a^{n}\right)$ is continuous on $\mathbb{R} \times \mathbb{R}^{\mathbf{n}}$

$\left.1^{\prime}\right) a(u, p) \cdot p \geq \nu_{0}|p|^{m}-\mu_{0}\left(\nu_{0}>0\right), \quad|a(u, p)| \leq \mu_{1}\left(|p|^{m-1}+1\right)$ for any $u \in \mathbb{R}, p \in \mathbb{R}^{n}$ 2' $^{\prime}(a(u, p)-a(u, q)) \cdot(p-q) \geq \nu_{1}|p-q|^{m}\left(\nu_{1}>0\right)$ for any $u \in \mathbb{R}, p, q \in \mathbb{R}^{n}$ $\left.\mathbf{3}^{\prime}\right)|a(u, p)-a(v, p)| \leq \Lambda|u-v|\left(|p|^{m-1}+1\right)(\Lambda \geq 0)$ for any $u, v \in \mathbb{R}$ and $p, q \in \mathbb{R}^{n}$ 4') $m>\max \left(1, \frac{2 n}{n+2}\right)$. 
Proposition 5.1. Let $f$ be measurable and bounded in $Q_{T}, \Psi \in \dot{W}_{2}^{1}\left(Q_{T}\right)$ and let conditions $\left.0^{\prime}\right)-4^{\prime}$ ) hold. Then the Cauchy-Dirichlet problem (5.10) has exactly one generalized (in sense of Definition 3.1) solution $u$. Moreover this solution belongs to $C\left([0, T] ; L_{2}(\Omega)\right)$.

Proof. Uniqueness of the generalized solution of problem (5.10) follows from Proposition 3.2. So we have to prove only existence of solution cited. The forthcoming proof is a suitable adaptation of the proof of Theorem 6.7 of [14: Chapter 5].

Let $\left\{\Psi_{k}\right\}_{k \in \mathbb{N}}$ be a basis in $\dot{W}_{m}^{1}(\Omega)$ such that $\int_{\Omega} \Psi_{k} \Psi_{l} d x=\delta_{k}^{l} \quad(k, l \in N)$, where $\delta_{k}^{l}$ is the Kronecker delta, and

$$
\sup \left(\left|\Psi_{k}\right|, \Omega\right)+\sup \left(\left|\nabla \Psi_{k}\right|, \Omega\right) \leq c_{k}=\text { const } \quad(k \in \mathbb{N}) .
$$

Set

$$
u^{\mathcal{N}}=\sum_{k=1}^{\mathcal{N}} c_{k}^{\mathcal{N}}(t) \Psi_{k}(x)
$$

where $\left\{c_{k}^{N}\right\}_{k=1, \ldots, N}$ is the solution of the system of ordinary differential equations

$$
\left(u_{i}^{N}, \Psi_{k}\right)+\left(a^{i}\left(u^{N}, \nabla u^{N}\right), \frac{\partial \Psi_{k}}{\partial x_{i}}\right)=\left(f, \Psi_{k}\right) \quad(k=1, \ldots, N)
$$

with initial conditions

$$
c_{k}^{N}(0)=\left(\Psi(x, 0), \Psi_{k}\right) \quad(k=1, \ldots, N) .
$$

From the conditions of Proposition 5.1 it follows that the second and third terms in (5.12) are bounded and measurable functions of the variables $t, c_{k}^{N}$ on any set $[0, T] \times$ $\left\{\left|c_{k}^{N}\right| \leq\right.$ const $\left.(k=1, \ldots, N)\right\}$; moreover these functions are continuous in $c_{k}^{N}$. Therefore existence at least of one solution of (5.12), (5.13) will be established if we could show that all possible solutions of this problem are uniformly bounded on $[0, T]$. Exactly in the same way as in [14: pp. $533-535]$ we can prove that the a priori estimate

$$
\sup _{t \in[0, T]}\left\|u^{N}\right\|_{L_{2}(\Omega)}^{2}+\left\|\nabla u^{N}\right\|_{L_{m}\left(Q_{T}\right)}^{m} \leq c
$$

holds with some constant $c$ independent of $N$. Then from (5.14) it follows that

$$
\sup _{t \in[0, T]} \sum_{k=1}^{N}\left|c_{k}^{N}(t)\right|^{2}=\sup _{t \in[0, T]}\left\|u^{N}\right\|_{L_{2}(\Omega)}^{2} \leq c
$$

and hence existence at least of one solution (5.12), (5.13) is established. ¿From (5.14) it follows (see [14: p. 534]) that

$$
\left\|u^{N}\right\|_{L_{m(n+2) / n}\left(Q_{T}\right)} \leq c
$$

where the constant $c$ is independent of $N$. Moreover, for any fixed $k$ the functions

$$
l_{N, k}(t)=\left(u^{N}(x, t), \Psi_{k}(x)\right) \quad(N, k \in \mathbb{N}) .
$$


are equicontinuous (with respect to $N)$ in't on $[0, T]$. Together with (5.14) it gives the possibility (see [14: p. 535]) to choose some subsequence $\left\{u^{N}\right\}$ that converges weakly in $L_{2}(\Omega)$ uniformly with respect to $t$ on $[0, T]$ to some function $u$ such that

$$
\sup \left(\|u\|_{L_{2}(\Omega)},[0, T]\right) \leq c .
$$

Moreover, using again (5.14) we can count that

$$
\frac{\partial u^{\mathcal{N}}}{\partial x_{i}} \longrightarrow \frac{\partial u}{\partial x_{i}} \quad \text { weakly in } L_{m}\left(Q_{T}\right) \text { as } N \rightarrow \infty
$$

and hence $u \in \stackrel{\circ}{W}_{m}^{1,0}\left(Q_{T}\right)$ and

$$
\|\nabla u\|_{L_{m}\left(Q_{T}\right)} \leq c
$$

with some constant $c$ depending only on the data (see [14: p. 535]).

Obviously, from (5.12) it follows that the integral identity

$$
\left.\int_{\Omega} u^{N} \varphi d x\right|_{0} ^{\tau}+\iint_{Q_{r}}\left(-u^{N} \varphi_{t}+a\left(u^{N}, \nabla u^{N}\right) \cdot \nabla \varphi\right) d x d t=\iint_{Q_{r}} f \varphi d x d t
$$

holds for any $\tau \in(0, T]$ and $\varphi=\sum_{k=1}^{N} d_{k}(t) \Psi_{k}(x)$ where $d_{k}$ are arbitrary functions continuous in $t$ on $[0, T]$ and having bounded on $[0, T]$ generalized derivatives $d_{k}^{\prime}$. Denote the class of such functions $\varphi$ as $\mathcal{P}_{\mathcal{N}}$. Obviously, $u^{N}$ belong to $\mathcal{P}_{\mathcal{N}}$. Denote $A_{N}^{i}=$ $a^{i}\left(u^{N}, \nabla u^{N}\right) \quad(i=1, \ldots, N)$. In view of the second inequality in condition $\left.1^{\prime}\right)$ and estimate (5.14) we have the uniform (with respect to $N$ ) estimate

$$
\left\|A_{i}^{N}\right\|_{L_{m^{\prime}}\left(Q_{r}\right)} \leq c \quad(i=1, \ldots, N ; N \in \mathbb{N})
$$

Therefore we can count that there exist functions $A_{i} \in L_{m^{\prime}}\left(Q_{T}\right)$ such that

$$
A_{i}^{N} \longrightarrow A_{i} \quad \text { weakly in } L_{m^{\prime}}\left(Q_{T}\right)
$$

Using estimate (5.14) and taking into account that $u^{N} \rightarrow u$ weakly in $L_{2}(\Omega)$ (uniformly with respect to $t$ on $[0, T])$ we derive from inequality $(5.2)$ in the case $\beta=0$ for the difference $u^{N}-u^{N_{1}}$ that

$$
u^{N} \longrightarrow u \quad \text { strongly in } L_{2, m}\left(Q_{T}\right)
$$

and hence we can count that

$$
u^{N} \longrightarrow u \quad \text { strongly in } L_{2}(\Omega) \text { for a.e. } t \in[0, T]
$$

and

$$
u^{N} \longrightarrow u \quad \text { a.e. in } Q_{T} \text {. }
$$

Moreover, in view of (5.16) and condition $\left.4^{\prime}\right)$

$$
u^{N} \longrightarrow u \quad \text { weakly in } L_{2}\left(Q_{T}\right) .
$$


Then from (5.21) and (5.23) - (5.27) we can conclude that for a.e. $\tau \in(0, T]$ and $\varphi \in \bigcup_{k=1}^{\infty} \mathcal{P}_{k}$

$$
\left.\int_{\Omega} u \varphi d x\right|_{0} ^{\tau}+\iint_{Q_{\tau}}\left(-u \varphi_{t}+A_{i} \varphi_{x_{i}}\right) d x d t=\iint_{Q_{\tau}} f \varphi d x d t
$$

In the same way as in [14: p. 538] we can derive from (5.28) and (5.18) that

$$
u \in C\left([0, T] ; L_{2}(\Omega)\right)
$$

and to prove that identity (5.28) holds for any $\tau \in(0, T]$. Moreover, we establish that for every $\tau \in(0, T]$

$$
\left.\frac{1}{2} \int_{\Omega} u^{2} d x\right|_{0} ^{r}+\iint_{Q_{r}} A_{i} u_{x_{i}} d x d t=\iint_{Q_{r}} f u d x d t .
$$

To prove that $u$ is a generalized solution of $(5.10)$ it is sufficient to establish that

$$
\iint_{Q_{r}} A_{i} \varphi_{x_{i}} d x d t=\iint_{Q_{r}} a^{i}(u, \nabla u) \varphi_{x_{i}} d x d t
$$

for any $\varphi \in \bigcup_{k=1}^{\infty} \mathcal{P}_{k}$ because $\bigcup_{k=1}^{\infty} \mathcal{P}_{k}$ is dense in $\stackrel{\circ}{m}_{m}^{1}\left(Q_{T}\right)$. To prove (5.31) it is sufficient to establish that

$$
\frac{\partial u^{N}}{\partial x_{i}} \longrightarrow \frac{\partial u}{\partial x_{i}} \quad(i=1, \ldots, N) \quad \text { a.e. in } Q_{\tau}
$$

because in view of (5.32) and (5.26), the continuity of the functions $a^{i}(u, p)$, condition $1^{\prime}$ ), estimate (5.14) and the Vitali theorem we obtain that for any $\varphi \in \bigcup_{k=1}^{\infty} \mathcal{P}_{k}$

$$
\lim _{N \rightarrow \infty} \iint_{Q_{r}} a^{i}\left(u^{N}, \nabla u^{N}\right) \varphi_{x_{i}} d x d t=\iint_{Q_{\tau}} a^{i}(u, \nabla u) \varphi_{x_{i}} d x d t
$$

On the other hand in view of (5.23)

$$
\lim _{N \rightarrow \infty} \iint_{Q_{\tau}} a^{i}\left(u^{N}, \nabla u^{N}\right) \varphi_{x_{i}} d x d t=\iint_{Q_{\tau}} A_{i} \varphi_{x_{i}} d x d t
$$

Hence (5.32) implies (5.31). The remainder of this section is devoted to proving of (5.32).

Choosing $\varphi=u^{N}$ in (5.21) we obtain

$$
\left.\frac{1}{2} \int_{\Omega}\left(u^{N}\right)^{2} d x\right|_{0} ^{\tau}+\iint_{Q,} a\left(u^{N}, \nabla u^{N}\right) \cdot \nabla u^{N} d x d t=\iint_{Q} f u^{N} d x d t
$$

Using (5.25) and (5.27) we derive from (5.33) and (5.30) that for any $\tau \in(0, T]$

$$
\lim _{N \rightarrow \infty} \iint_{Q_{r}} a\left(u^{N}, \nabla u^{N}\right) \cdot \nabla u^{N} d x d t=\iint_{Q_{r}} A_{i} u_{x_{i}} d x d t
$$


Using now condition $2^{\prime}$ ) we have

$$
\begin{aligned}
& \nu_{1} \iint_{Q_{r}}\left|\nabla u^{N}-\nabla u\right|^{m} d x d t \\
& \quad \leq \iint_{Q_{r}}\left(a\left(u^{N}, \nabla u^{N}\right)-a\left(u^{N}, \nabla u\right)\right) \cdot\left(\nabla u^{N}-\nabla u\right) d x d t .
\end{aligned}
$$

Using (5.19), (5.23) and (5.34) and taking into account (in view of $\left.1^{\prime}\right),(5.20)$ and (5.26)) that

$$
a^{i}\left(u^{N}, \nabla u\right) \longrightarrow a^{i}(u, \nabla u) \quad \text { strongly in } L_{m^{\prime}}\left(Q_{T}\right) \text { as } N \rightarrow \infty
$$

we derive from (5.35)

$$
\lim _{N \rightarrow \infty} \iint_{Q_{r}}\left|\nabla u^{N}-\nabla u\right|^{m} d x d t=0
$$

But from (5.37) it follows that (5.32) holds for some subsequence $\left\{u^{N}\right\}$

\section{A priori estimates for solutions of regularized Cauchy-Dirichlet problems}

In view of Theorem 3.1 to prove Theorem 1.1 it is sufficient to establish the following

Theorem 6.1. Let conditions $(\Omega),(\mathrm{BI}),(\mathrm{RHS})$ and 0$)$ - 4) hold. Then the CauchyDirichlet problem

(CD) $F[u]=f$ in $Q_{T}$

$$
u=\Psi \text { on } \Gamma T
$$

has at least one regular (in sense of Definition 2.5) solution.

The result of Theorem 6.1 correspondent to the case

$$
m \geq 2 \quad \text { and } \quad l \geq 0
$$

can be derived from the proof of the main theorem of the paper [11] if to use Theorem 4.1 of the present paper. Therefore we shall prove Theorem 6.1 only in the case when

$$
1<m<2 \quad \text { and } \quad l \geq 0 .
$$

It is easy to see that

$$
\omega \subset\{(m, l): 1<m<2\} \times\{(m, l): l \geq 0\} .
$$

The proof of Theorem 6.1 correspondent to the case (6.2) can be easily transformed in one applicable in the case (6.1). 
In the remainder of this paper we assume that all conditions $(\Omega),(\mathrm{BI}),(\mathrm{RHS})$ and $0)$ - 4) of Theorem 6.1 and also condition (6.2) are fulfilled.

Consider the regularized Cauchy-Dirichlet problems

$$
\begin{array}{cl}
(\mathbf{R C D})_{\delta, e, N} & F_{\delta, \varepsilon, N}[u]:=\frac{\partial u}{\partial t}-\delta \nabla u-\operatorname{div} a(\chi(u), \nabla u)=f \text { in } Q_{T} \\
& u=\Psi+\varepsilon \text { on } \Gamma_{T}
\end{array}
$$

where

$$
\delta>0, \quad \chi(u)=\min \{\max (u, \varepsilon), N\}, \quad \varepsilon>0, \quad N>\varepsilon .
$$

Without loss of generality we can and shall count that $\delta \leq 1$ and $\varepsilon \leq 1$. It is easy to see that in view of conditions 0 ) - 4) and (6.2) and the structure of the left-hand side of equation in $(\mathrm{RCD})_{\delta, \varepsilon, N}$ assumptions $\left.0^{\prime}\right)-3^{\prime}$ ) of Proposition 5.1 are fulfilled with $m=2$ because $\varepsilon \leq \chi(u) \leq N$ and $|p|^{m-1}+1 \leq|p|+1$ for any $m \in(1,2)$.

Denote $v=u-\varepsilon$ and consider the Cauchy-Dirichlet problem

$$
\begin{aligned}
\frac{\partial v}{\partial t}-\delta \nabla v-\operatorname{div} a(\chi(v+\varepsilon), \nabla v) & =f & \text { in } \quad Q_{T} \\
v & =\Psi & \text { on } \Gamma_{T}
\end{aligned}
$$

where $\Psi \in \stackrel{\circ}{W}_{2}^{1}\left(Q_{T}\right)$. In view of previous conclusions it follows obviously that for the problem (6.4) all conditions of Proposition 5.1 are fulfilled with $m=2$. Hence there exists exactly one generalized solution $v$ of this problem (such that $v \in C\left([0, T] ; L_{2}(\Omega)\right) \cap$ $\left.\stackrel{\circ}{W}_{2}^{1}\left(Q_{T}\right)\right)$. But then the Cauchy-Dirichlet problem $(\mathrm{RCD})_{\delta, \varepsilon, N}$ has exactly one generalized solution $u$ such that $u \in C\left([0, T] ; L_{2}(\Omega)\right) \cap W_{2}^{1,0}\left(Q_{T}\right)$, i.e., we proved the following

Lemma 6.1. For any $\delta>0, \varepsilon>0, N>\varepsilon$ the Cauchy-Dirichlet problem $(\mathrm{RCD})_{\delta, \varepsilon, N}$ has exactly one generalized solution $u \in C\left([0, T] ; L_{2}(\Omega)\right) \cap W_{2}^{1,0}\left(Q_{T}\right)$.

In the remainder of this section we consider problem $(\mathrm{RCD})_{\delta, \varepsilon, N}$ for $\delta \geq 0, \varepsilon>0$ and $N>\epsilon$. Now the term "generalized solution $u$ " means in particular that $u \in$ $C\left([0, T] ; L_{2}(\Omega)\right) \cap W_{2}^{1,0}\left(Q_{T}\right)$ in the case $\delta>0$ and $u \in C\left([0, T] ; L_{2}(\Omega)\right) \cap W_{m}^{1,0}\left(Q_{T}\right)$ in the case $\delta=0$.

Lemma 6.2. Let $u$ be a generalized solution of problem (RCD) $\delta_{,, \varepsilon, N}$ for any fixed $\delta \geq 0, \varepsilon>0$ and $N>\varepsilon$. Then

$$
\inf \left(u, Q_{T}\right) \geq \varepsilon
$$

Proof. Obviously that the conditions of Theorem 6.1 imply validity of the assumptions (G), (M) and (L) of Proposition 3.1 for the operator $F_{\delta, \varepsilon, N}[u]$ (with $m=2$ if $\delta>0)$. Then taking into account that $F_{\delta, \varepsilon, N}[u]=f, F_{\delta, e, N}[\varepsilon]=0$ and $u=\varepsilon$ on $S_{T}$, we can apply Proposition 3.1 for $u_{1}=\varepsilon, u_{2}=u$ and $f_{1}=0, f_{2}=f$. Using that $u_{1}=\varepsilon \leq \Psi+\varepsilon=u_{2}$ on $\Omega \times\{t=0\}$ (because $\Psi \geq 0$ ) we derive from (3.2) that $(\varepsilon-u)^{+} \leq 0$ a.e. in $Q_{T}$, i.e., $u \geq \varepsilon$ a.e. in $Q_{T}$ 
Lemma 6.3. There exist constants $c_{1}$ and $c_{2}$ depending on $n, m, l$, the parameters from conditions 1) - 3), and $\sup \left(\Psi, \bar{Q}_{T}\right)$ such that for any generalized solution $u$ of problem $(\mathrm{RCD})_{\delta, \varepsilon, N}$ with any fixed $\delta \geq 0, \varepsilon>0$ and $N \geq c_{1}$ we have

$$
\sup \left(u, Q_{T}\right) \leq c_{1}
$$

and

$$
\varepsilon^{l} \iint_{Q_{T}}|\nabla u|^{m} d x d t \leq \iint_{Q_{T}}\left|\nabla u^{\alpha+1}\right|^{m} d x d t \leq c_{2} \quad\left(\alpha=\frac{l}{m}\right) .
$$

Proof. The proof of validity of estimates (6.6) and (6.7) in the case $m+l \geq 2$ is given in [11]. The case $m+l<2$ required to find new (a more difficult) version of the Moser method of establishing $L_{\infty}$-estimates. It was made in paper [10]. In the case $m+l<2$ the lemma follows from Theorems 1.1 and 1.2 of [10]

Remark 6.1. In the remainder of this paper we consider problem $(\mathrm{RCD})_{\delta, e, N}$ with $N=c_{1}$ where the constant $c_{1}$ is defined by Lemma 6.3. In view of estimates (6.5) and (6.6) we can rewrite problem $(\mathrm{RCD})_{\delta, \varepsilon, N}$ as

$$
\begin{aligned}
(\mathbf{R C D})_{\delta, \varepsilon} & F_{\delta, \varepsilon}[u]:=\frac{\partial u}{\partial t}-\delta \nabla u-\operatorname{div} a(u, \nabla u)=f \text { in } Q_{T} \\
& u=\Psi+\varepsilon \text { on } \Gamma_{T}
\end{aligned}
$$

where $\delta>0$ and $\varepsilon>0$.

Lemma 6.4. Let $u$ be a generalized solution of the Cauchy-Dirichlet problem $(\mathrm{RCD})_{\delta, \varepsilon}$ for $\delta=0$ and $\varepsilon>0$. Then there exist constants $\lambda \in(0,1)$ and $K>0$ independent of $\varepsilon$ such that (see (4.21))

$$
\langle u\rangle_{\lambda, \bar{Q}_{T}} \leq K
$$

Proof. In view of conditions 1) - 3), Remark 6.1, estimates (6.5) - (6.7) and Remark 4.1 we can apply either Theorem 4.1 or Theorem 4.2 and hence establish (6.8) with some $\lambda \in(0,1)$ and $K>0$ independent of $\varepsilon$

\section{The passing to the limit as $\delta \rightarrow 0$}

In this section we show that generalized solutions $u_{\delta}$ of the Cauchy-Dirichlet problems $(\mathrm{RCD})_{\delta, \varepsilon}($ for any fixed $\varepsilon>0$ ) tend to a generalized solution of the Cauchy-Dirichlet problem

$$
\begin{array}{cl}
(\mathbf{R C D})_{\epsilon} & F_{\varepsilon}[u]:=\frac{\partial u}{\partial t}-\operatorname{div} a(u, \nabla u)=f \text { in } Q_{T} \\
& u=\Psi+\varepsilon \text { on } \Gamma_{T}
\end{array}
$$

as $\delta \rightarrow 0$. For proving this we use estimates (6.5) - (6.7) and Lemma 5.1 with appropriate $\beta>0$. 
Obviously, the functions $u_{\delta}$ satisfy for any $\tau \in(0, T]$ and every function $\phi \in \stackrel{\circ}{W}_{2}^{1}\left(Q_{T}\right)$ the integral identity

$$
\left.\int_{\Omega} u_{\delta} \phi d x\right|_{0} ^{\tau}+\iint_{Q_{T}}\left(-u_{\delta} \psi+\delta \nabla u_{\delta} \cdot \nabla \phi+a\left(u_{\delta}, \nabla u_{\delta}\right) \cdot \nabla \phi-f \phi\right) d x d t=0
$$

Set here $\phi=\Psi \in C_{0}^{1}(\Omega)$. Then from condition 1) and estimates (6.6) and (6.7) it follows that for any $t_{1}, t_{2} \in[0, T]$ we have

$$
\begin{aligned}
\left|\int_{\Omega} u_{\delta} \Psi d x\right|_{t_{1}}^{t_{2}} \mid & \leq c \int_{t_{1}}^{t_{2}}\left(\left|\nabla u_{\delta}\right|+\left|\nabla u_{\delta}^{\alpha+1}\right|^{m-1}+1\right) d x d t \\
& \leq c\left(\left(\left|t_{2}-t_{1}\right||\Omega|\right)^{1 / 2}+\left(\left|t_{2}-t_{1}\right||\Omega|\right)^{1 / m}+\left|t_{2}-t_{1}\right||\Omega|\right) .
\end{aligned}
$$

From here it follows that the integrals $\int_{\Omega} u_{\delta} \Psi d x \quad(\delta \in(0,1))$ are equicontinuous (with respect to $\delta$ ) in $t$ on $[0, T]$ for any fixed $\Psi \in C_{0}^{1}(\bar{\Omega})$. Using the density of $C_{0}^{1}(\bar{\Omega})$ in $L_{2}(\Omega)$ and the uniform boundedness of the sequence $\left\{u_{\delta}\right\}$ in $Q_{T}$ (see (6.6)) we can derive from here that there exists a subsequence $\left\{u_{\delta}\right\}$ which converges weakly in $L_{2}(\Omega)$, uniformly with respect to $t$ on $[0, T]$, to some function $u$ satisfying inequality $(5.18)$ with a constant $c$ independent of $\delta$ (see also [14: pp. 182-183]). Moreover, in view of $(6.5)-(6.7)$ we can count that

$$
\begin{aligned}
& \nabla u_{\delta}^{\alpha+1} \longrightarrow \nabla u^{\alpha+1} \quad \text { weakly in } L_{m}\left(Q_{T}\right) \text { as } \delta \rightarrow 0 \\
& \nabla u_{\delta} \rightarrow \nabla u \quad \text { weakly in } L_{m}\left(Q_{T}\right) \text { as } \delta \rightarrow 0 \\
& \sqrt{\delta} \nabla u_{\delta} \longrightarrow 0 \quad \text { weakly in } L_{2}\left(Q_{T}\right) \text { as } \delta \rightarrow 0 \\
& \sup \left(u_{\delta}, Q_{T}\right)+\sup \left(u, Q_{T}\right) \leq c_{1}
\end{aligned}
$$

and

$$
\begin{aligned}
& \varepsilon^{l} \iint_{Q_{T}}\left|\nabla u_{\delta}\right|^{m} d x d t+\varepsilon^{l} \iint_{Q_{T}}|\nabla u|^{m} d x d t \\
& \quad+\iint_{Q_{T}}\left|\nabla u_{\delta}^{\alpha+1}\right|^{m} d x d t+\iint_{Q_{T}}\left|\nabla u^{\alpha+1}\right|^{m} d x d t \leq c_{2}
\end{aligned}
$$

where $\alpha=\frac{1}{m}$. Denote $A_{\delta}^{i}=a^{i}\left(u_{\delta}, \nabla u_{\delta}\right) \quad(i=1, \ldots, n)$. In view of condition 1) and inequalities (7.6) and (7.7) we have the estimate uniform with respect to $\delta$

$$
\left\|A_{\delta}^{i}\right\|_{L_{m^{\prime}}\left(Q_{T}\right)} \leq c \quad(i=1, \ldots, n ; \delta>0) .
$$

Then we can count that there exist functions $A^{i} \in L_{m^{\prime}}\left(Q_{T}\right)(i=1, \ldots, n)$ such that

$$
A_{\delta}^{i} \longrightarrow A^{i} \text { weakly in } L_{m^{\prime}}\left(Q_{T}\right) \text { as } \delta \rightarrow 0 \quad(i=1, \ldots, n) .
$$

On the other hand, from inequalities (7.6) and (7.7) it follows that for any $\delta, \delta^{\prime}>0$

$$
\iint_{Q_{T}}\left|\nabla\left(\left|u_{\delta}-u_{\delta^{\prime}}\right|^{\beta}\left(u_{\delta}-u_{\delta^{\prime}}\right)\right)\right|^{m} d x d t \leq c \quad\left(\beta=\frac{\sigma}{\sigma+2}\right)
$$


with some constant $c$ independent of $\delta$. Really, in view of the definition of $\beta$ we have $\frac{\beta+1}{2}=\frac{\sigma+1}{\sigma+2}$ and hence the conditions $m>1, \frac{1}{m}<\frac{1}{n}+\frac{1+\beta}{2}$ of Lemma 5.3 are fulfilled for $\beta=\frac{\sigma}{\sigma+2}$ in view of condition 4). It is easy to see that from inequalities (7.6) and (7.7) it follows that the constant $c$ in (7.10) is independent of $\delta$. Using (7.10) and taking into account that $u_{\delta} \rightarrow u$ weakly in $L_{2}(\Omega)$, uniformly with respect to $t$ on $[0, T]$, we derive from inequality (5.2) in the case $\beta=\frac{\sigma}{\sigma+2}$ for the difference $u_{\delta}-u_{\delta^{\prime}}$ that

$$
\begin{array}{ll}
u_{\delta} \longrightarrow u & \text { strongly in } L_{2, m}\left(Q_{T}\right) \\
u_{\delta} \longrightarrow u & \text { a.e. in } Q_{T} \\
u_{\delta} \longrightarrow u & \text { weakly in } L_{2}\left(Q_{T}\right) \\
u_{\delta} \longrightarrow u & \text { strongly in } L_{2}(\Omega) \text { for a.e. } t \in[0, T]
\end{array}
$$

Then from (7.2), (7.5), and (7.12) - (7.14) we can derive that for a.e. $\tau \in(0, T)$ and any $\phi \in \stackrel{\circ}{W}_{2}^{1}\left(Q_{T}\right)$

$$
\left.\int_{\Omega} u \phi d x\right|_{0} ^{r}+\iint_{Q_{r}}\left(-u \phi_{t}+A^{i} \phi_{x_{i}}-f \phi\right) d x d t=0 .
$$

The following proposition is well-known (see, for example, [11]).

Proposition 7.1. Let the function $g$ satisfy a Lipschitz condition uniformly on $\mathbb{R}$ and its derivative $g^{\prime}$ be continuous everywhere on $\mathbb{R}$ with possible exception of finitely many points at which $g^{\prime}$ has a discontinuity of the first order. Further, let $u \in C\left([0, T] ; L_{2}(\Omega)\right) \cap W_{m}^{1,0}\left(Q_{T}\right), \varphi \in W_{m}^{1}\left(Q_{T}\right), f_{i} \in L_{m^{\prime}}\left(Q_{T}\right) \quad\left(i=0,1, \ldots, n\right.$, $\frac{1}{m}+$ $\left.\frac{1}{m^{\prime}}=1(m>1)\right)$. At least, assume that for any $t_{1}, t_{2} \in[0, T]$ and any $\phi \in \dot{W}_{m}^{1}\left(Q_{T}\right)$

$$
\left.\int_{\Omega} u \phi d x\right|_{t_{1}} ^{t_{2}}+\int_{t_{1}}^{t_{2}} \int_{\Omega}\left(-u \phi_{t}+f_{i} \phi_{x_{i}}+f_{0} \phi\right) d x d t=0
$$

and let $u=\varphi$ on $S_{T}$. Then for any $t_{1}, t_{2} \in[0, T]$ we have

$$
\begin{aligned}
& \left.\int_{\Omega}(G(u)-u g(\varphi)) d x\right|_{t_{1}} ^{t_{2}} \\
& \quad+\int_{t_{1}} \int_{\Omega}\left(u g^{\prime}(\varphi) \varphi_{t}+f_{i}\left(g^{\prime}(u) u_{x_{i}}-g^{\prime}(\varphi) \varphi_{x_{i}}\right)+f_{0}(g(u)-g(\varphi))\right) d x d t \\
& =0
\end{aligned}
$$

where $G(u)=\int_{0}^{u} g(\xi) d \xi$.

Using Proposition 7.1 we can conclude (in the same way as in [14: p. 538]) that in view of (7.15) and (5.18) or (7.6) condition (5.29) holds for the function $u$. Moreover, using Proposition 7.1 we can derive from (7.15) that for any $\tau \in(0, T]$ we have

$$
\left.\int_{\Omega}\left(\frac{1}{2} u^{2}-u \varepsilon\right) d x\right|_{0} ^{r}+\iint_{Q_{r}}\left(A^{i} u_{x_{i}}-f(u-\varepsilon)\right) d x d t=0 .
$$


In view of (5.29) the integral identity (7.15) holds for any $\tau \in(0, T]$.

To prove that $u$ is a generalized solution of problem $(\mathrm{RCD})_{\epsilon}$ it is sufficient to establish that

$$
\iint_{Q_{r}} A^{i} \phi_{x_{i}} d x d t=\iint_{Q_{r}} a^{i}(u, \nabla u) \phi_{x_{i}} d x d t
$$

for any $\phi \in C_{0}^{1}(\Omega)$ (because $C_{0}^{1}(\Omega)$ is dense in $\dot{W}_{m}^{1}\left(Q_{T}\right)$ ). To prove this it is sufficient to establish that

$$
\frac{\partial u_{\delta}}{\partial x_{i}} \longrightarrow \frac{\partial u}{\partial x_{i}} \quad \text { a.e. in } Q_{T} \quad(i=1, \ldots, n)
$$

because in view of (7.19) and (7.12), the continuity of the functions $a^{i}$, condition $1^{\prime}$ ), estimates (7.6) and (7.7) and the Vitali theorem we obtain that for any $\phi \in W_{m}^{1}\left(Q_{T}\right)$ and any $\tau \in(0, T]$

$$
\lim _{\delta \rightarrow 0} \iint_{Q_{r}} a^{i}\left(u_{\delta}, \nabla u_{\delta}\right) \phi_{x_{i}} d x d t=\iint_{Q_{r}} a^{i}(u, \nabla u) \phi_{x_{i}} d x d t
$$

On the other hand, in view of (7.9) the left-hand side here is equal to that of (7.18). Hence (7.19) implies (7.18).

Choosing $\phi=u_{\delta}-\varepsilon$ in (7.1) we obtain with the aid of Proposition 7.1 that

$$
\left.\int_{\Omega}\left(\frac{1}{2} u_{\delta}^{2}-u_{\delta} \varepsilon\right) d x\right|_{0} ^{\tau}+\iint_{Q_{r}}\left(a^{i}\left(u_{\delta}, \nabla u_{\delta}\right) \frac{\partial u_{\delta}}{\partial x_{i}}-f\left(u_{\delta}-\varepsilon\right)\right) d x d t=0 .
$$

Using (7.13) and (7.14) we derive from (7.21) and (7.17) that for any $\tau \in(0, T]$

$$
\lim _{\delta \rightarrow 0} \iint_{Q_{r}} a^{i}\left(u_{\delta}, \nabla u_{\delta}\right) \frac{\partial u_{\delta}}{\partial x_{i}} d x d t=\iint_{Q_{r}} A^{i} \frac{\partial u}{\partial x_{i}} d x d t .
$$

Using now condition 2) we have

$$
\begin{aligned}
\nu_{1} J_{\delta}: & =\nu_{1} \iint_{Q_{r}} \frac{\left|\nabla u_{\delta}-\nabla u\right|^{2}}{\left(\left|\nabla u_{\delta}\right|^{m}+|\nabla u|^{m}\right)^{2 / m-1}} d x d t \\
& \leq \iint_{Q_{r}}\left(a\left(u_{\delta}, \nabla u_{\delta}\right)-a(u, \nabla u)\right) \cdot\left(\nabla u_{\delta}-\nabla u\right) d x d t \\
& =: \mathcal{H}_{\delta} .
\end{aligned}
$$

Using (7.3) - (7.5), (7.9) and (7.22) and taking into account that in view of (7.12), (7.6) and (7.7), condition 1) and the Vitali theorem

$$
a^{i}\left(u_{\delta}, \nabla u\right) \longrightarrow a^{i}(u, \nabla u) \text { strongly in } L_{m^{\prime}}\left(Q_{T}\right) \text { as } \delta \rightarrow 0
$$

we derive from (7.23) that

$$
\lim _{\delta \rightarrow 0} \mathcal{H}_{\delta}=0
$$


Using this limit and inequalities $0 \leq J_{\delta} \leq \nu_{1}^{-1} \mathcal{H}_{\delta}$ we obtain

$$
\lim _{\delta \rightarrow 0} J_{\delta}=0
$$

Show that from here it follows that (7.19) is true. Denote

$$
h_{\delta}(x, t):=\frac{\left|\nabla u_{\delta}-\nabla u\right|^{2}}{\left(\left|\nabla u_{\delta}\right|^{m}+|\nabla u|^{m}\right)^{2 / m-1}}
$$

From $\lim _{\delta \rightarrow 0} J_{\delta}=0$ it follows that there exist some subsequence $\{\delta\}$ and subset $\widetilde{Q} \subset$ $Q_{\tau},|\widetilde{Q}|=\left|Q_{r}\right|$, such that

$$
\lim _{\delta \rightarrow 0} h_{\delta}(x, t)=0 \quad \text { on } \widetilde{Q}
$$

Without loss of generality we can count that $\frac{\partial u}{\partial x_{i}}$ are finite on $\tilde{Q}$, i.e., $|\nabla u|$ is bounded (non-uniformly) at any point $(x, t) \in \widetilde{Q}$. In view of $(7.27)$ we have for any $(x, t) \in \widetilde{Q}$

$$
h_{\delta}(x, t) \geq \frac{\left(\left|\nabla u_{\delta}\right|-c\right)^{2}}{\left(\left|\nabla u_{\delta}\right|+c\right)^{2-m}}
$$

with a constant $c$ depending on $(x, t) \in \widetilde{Q}$. Suppose now that $\left|\nabla u_{\delta}\right|$ is unbounded in some point $(x, t) \in \widetilde{Q}$. Then $\left|\nabla u_{\delta}\right| \rightarrow \infty$ for some subsequence $\{\delta\}$ and hence in view of (7.29) we obtain that for this subsequence $\lim _{\delta \rightarrow \infty} h_{\delta}(x, t)=\infty$, i.e., we obtain a contradiction with (7.28). Hence

$$
\left|\nabla u_{\delta}\right| \quad \text { are bounded (non-uniformly) at any point of } \tilde{Q} \text {. }
$$

Then from (7.27), (7.28) and (7.30) it follows that the numerators of $h_{\delta}$ tend to zero on $\widetilde{Q}$ as $\delta \rightarrow 0$, i.e. (7.19) is true. Therefore the function $u \in C\left([0, T] ; L_{2}(\Omega)\right) \cap W_{m}^{1,0}\left(Q_{T}\right)$ is a generalized solution of problem $(\mathrm{RCD})_{\varepsilon}$. From Lemmas 6.2 and 6.3 it follows that this function satisfies estimates (6.5) - (6.8). In view of (6.5) and Proposition 3.3 the function $u$ is a unique strong solution of problem (RCD) $)_{e}$. So we proved the following

Lemma 7.1. For any fixed $\varepsilon>0$ there exist exactly one strong solution (in sense of Definition 2.3) of problem (RCD)e satisfying estimates (6.5) - (6.8) with constants $c_{1}, c_{2}, \lambda \in(0,1)$ and $K$ independent of $\varepsilon$. 


\section{The passing to the limit as $\varepsilon \rightarrow 0$}

Now we are ready to prove Theorem 6.1 and hence Theorem 1.1. In the remainder of this section we denote the solution of problem $(\mathrm{RCD})_{\varepsilon}$ as $u_{\epsilon}$. We are going to realize the passing to the limit as $\varepsilon \rightarrow 0$ using the a priori estimates (6.5) - (6.8). This passing can be done in the same way as one in [12] where existence of regular solution of problem (CD) was proved in the case $l \geq 0, \max \left(1, \frac{2 n}{n+2}\right)<m<2, m+l \geq 2$. that

In view of estimates (6.5) - (6.8) we can conclude that there exists a function $u$ such

$$
\begin{aligned}
& u_{\varepsilon} \rightarrow u \quad \text { uniformly in } Q_{T} \\
& \frac{\partial}{\partial x_{i}} u_{\varepsilon}^{\alpha+1} \longrightarrow u^{\alpha} u_{x_{i}} \quad \text { weakly in } L_{m}\left(Q_{T}\right) \quad\left(i=1, \ldots, n ; \alpha=\frac{l}{m}\right) \\
& 0 \leq \inf \left(u, Q_{T}\right) \leq \sup \left(u, Q_{T}\right) \leq c_{1} \\
& \iint_{Q_{T}} u^{\prime}\left|u_{x}\right|^{m} d x d t \leq c_{2}
\end{aligned}
$$

and (see (4.2))

$$
\langle u\rangle_{\lambda, \bar{Q}_{T}} \leq K
$$

In (8.2) and (8.4) we used the following notation similar to one from Definition 2.3:

$$
\begin{aligned}
& u_{x}=\left(u_{x_{1}}, \ldots, u_{x_{n}}\right) \\
& u_{x_{i}}=\left\{\begin{array}{ll}
(\alpha+1)^{-1} u^{-\alpha} & \text { on }\left\{Q_{T}: u>0\right\} \\
0 & \text { on }\left\{Q_{T}: u=0\right\}
\end{array} \quad\left(\alpha=\frac{l}{m}\right) .\right.
\end{aligned}
$$

Obviously, $u^{\alpha} u_{x_{i}} \in L_{m}\left(Q_{T}\right) \quad(i=1, \ldots, n)$ (in view of (8.4)). In view of the boundedness of $u$ and inequality $\sigma=\frac{l}{m-1}>\alpha$ the expressions for $u_{x_{i}}$ in (8.6) and (2.2) coincide. Moreover, from condition $u^{\alpha} u_{x_{i}} \in L_{m}\left(Q_{T}\right)$ it follows that $u^{\sigma} u_{x_{i}} \in L_{m}\left(Q_{T}\right)$ (i= $1, \ldots, n)$. We use below the following auxiliary propositions (see [11] or [12]).

Proposition 8.1. Let the function $u$ be bounded and non-negative in $Q_{T}$ and such that $\nabla u^{\alpha+1} \in L_{m}\left(Q_{T}\right)$ for some $\alpha \geq 0$. Further, let the function $\bar{u}$ be defined by

$$
\bar{u}=\sup \left(u-\varepsilon_{1}, 0\right) \quad\left(\varepsilon_{1}=\text { const }>0\right) .
$$

Then $\bar{u}$ has generalized derivatives $\frac{\partial \bar{u}}{\partial x_{i}} \in L_{m}\left(Q_{T}\right)(i=1, \ldots, n)$ such that

$$
\frac{\partial \bar{u}}{\partial x_{i}}= \begin{cases}u_{x_{i}} & \text { in }\left\{Q_{T}: u>\varepsilon_{1}\right\} \\ 0 & \text { in }\left\{Q_{T}: 0 \leq u \leq \varepsilon_{1}\right\}\end{cases}
$$

where $u_{x_{i}}$ are defined by (8.6). Moreover,

$$
\lim _{\varepsilon_{1} \rightarrow 0}\left\|u^{\alpha} \frac{\partial \bar{u}}{\partial x_{i}}-u^{\alpha} u_{x_{i}}\right\|_{L_{m}\left(Q_{T}\right)}=0 .
$$


Proposition 8.2. Let $A^{i} \in L_{m^{\prime}}\left(Q_{T}\right) \quad(i=1, \ldots, n)$ and $B \in L_{m^{\prime}}\left(Q_{T}\right) \quad\left(\frac{1}{m}+\right.$ $\left.\frac{1}{m^{\prime}}=1, m>1\right)$, and let the function $u$ be bounded and non-negative in $Q_{T}$ and such that $\nabla u^{\alpha+1} \in L_{m}\left(Q_{T}\right)$ for some $\alpha \geq 0$. Assume that for any $t_{1}, t_{2} \in[0, T]$ and any $\phi \in \dot{W}_{m}^{1}\left(Q_{T}\right)$

$$
\left.\int_{\Omega} u \phi d x\right|_{t_{1}} ^{t_{2}}+\int_{t_{1}}^{t_{2}} \int_{\Omega}\left(-u \phi_{t}+u^{\alpha} A^{i} \phi_{x_{i}}+B \phi\right) d x d t=0
$$

Let $\varphi \in W_{m}^{1}\left(Q_{T}\right)$ and $u=\varphi$ on $S_{T}$. Then for any $t_{1}, t_{2} \in[0, T]$

$$
\left.\int_{\Omega}\left(\frac{1}{2} u^{2}-u \varphi\right) d x\right|_{t_{1}} ^{t_{2}}+\int_{t_{1}}^{t_{2}} \int_{\Omega}\left(u \varphi_{t}+u^{\alpha} A^{i}\left(u_{x_{i}}-\varphi_{x_{i}}\right)+B(u-\varphi)\right) d x d t=0
$$

where $u_{x_{i}}$ are defined by (8.8).

Returning to (8.1) - (8.5) we see that the function $u$ is non-negative and bounded in $Q_{T}, u \in C^{\lambda, \lambda / m}\left(\bar{Q}_{T}\right), \nabla u^{\alpha+1} \in L_{m}\left(Q_{T}\right) \quad\left(\alpha=\frac{l}{m}\right)$ (so that $\nabla u^{\sigma+1} \in L_{m}\left(Q_{T}\right), \sigma=$ $\left.\frac{l}{m-1}\right)$ and $u=\psi$ on $\Gamma_{T}$. Hence to prove Theorem 6.1 it is sufficient to show that for any $t_{1}, t_{2} \in[0, T]$ and $\phi \in \stackrel{\circ}{W}_{m}^{1}\left(Q_{T}\right)$

$$
\left.\int_{\Omega} u \phi d x\right|_{t_{1}} ^{t_{2}}+\int_{t_{1}}^{t_{2}} \int_{\Omega}\left(-u \phi_{t}+a\left(u, u_{x}\right) \cdot \nabla \phi-f \phi\right) d x d t=0
$$

where $u_{x}$ is defined by (8.8) in the case $\alpha=\frac{l}{m}$. Really, in this case from the kind of the problem $(\mathrm{RCD})_{\epsilon}$ it will follow that $u$ is a quasistrong and hence regular solution of problem (CD).

To prove that (8.12) holds denote

$$
A_{\varepsilon}^{i}=u_{e}^{-\alpha} a^{i}\left(u_{e}, \nabla u_{e}\right) \quad\left(\alpha=\frac{l}{m} ; i=1, \ldots, n\right) .
$$

In view of the second inequality in condition 1) and estimate (8.4) we have the uniform estimate

$$
\left\|A_{\varepsilon}^{i}\right\|_{L_{m^{\prime}}(Q r)} \leq c \quad(i=1, \ldots, n) .
$$

Then we can count that there exist functions $a^{i} \in L_{m^{\prime}}\left(Q_{T}\right)$ such that

$$
A_{\varepsilon}^{i} \longrightarrow A^{i} \quad \text { weakly in } L_{m^{\prime}}\left(Q_{T}\right) \text { as } \varepsilon \rightarrow 0 \quad(i=1, \ldots, n) .
$$

Letting $\varepsilon \rightarrow 0$ in the integral identity

$$
\left.\int_{\Omega} u_{\varepsilon} \phi d x\right|_{t_{1}} ^{t_{2}}+\int_{t_{1}}^{t_{2}} \int_{\Omega}\left(-u_{e} \phi_{t}+a\left(u_{e}, \nabla u_{e}\right) \cdot \nabla \phi-f \phi\right) d x d t=0
$$


for $\phi \in \dot{W}_{m}^{1}\left(Q_{T}\right)$ we obtain in view of (8.1) and (8.15) that for any $t_{1}, t_{2} \in[0, T]$ and $\phi \in \stackrel{W}{m}_{m}^{1}\left(Q_{T}\right)$

$$
\left.\int_{\Omega} u \phi d x\right|_{t_{1}} ^{t_{2}}+\int_{i_{1}}^{t_{2}} \int_{\Omega}\left(-u \phi_{t}+u^{\alpha} A^{i} \phi_{x_{i}}-f \phi\right) d x d t=0
$$

To prove Theorem 6.1 it is sufficient to show that

$$
\int_{i_{1}}^{t_{2}} \int_{\Omega} u^{\alpha} A^{i} \phi_{x_{i}} d x d t=\int_{i_{1}}^{t_{2}} \int_{\Omega} a^{i}(u, \nabla u) \phi_{x_{i}} d x d t=0 \quad \text { for any } \phi \in \dot{C}\left(\bar{Q}_{T}\right)
$$

because $A^{i}, a^{i}(u, \nabla u) \in L_{m^{\prime}}\left(Q_{T}\right)$ and $\dot{C}^{1}\left(\bar{Q}_{T}\right)$ is dense in $\dot{W}_{m}^{1}\left(Q_{T}\right)$. To prove equality $(8.18)$ it is sufficient to establish that for some subsequence $\{\varepsilon\}$

$$
u_{\varepsilon}^{\alpha} \frac{\partial u_{\varepsilon}}{\partial x_{i}} \longrightarrow u^{\alpha} u_{x_{i}} \quad \text { a.e. in } Q_{T} \quad(i=1, \ldots, n)
$$

because in view of (8.19) and (8.1), the continuity of the functions $u^{-\alpha} a^{i}\left(u, u^{-\alpha} p\right)(\alpha=$ $\frac{l}{m}$ ) on $\overline{\mathbb{R}}_{+} \times \mathbb{R}^{n}$, condition 1 ), the uniform estimate (6.7) for $u=u_{\varepsilon}$ and the Vitali theorem we obtain that for any $\phi \in \stackrel{\circ}{C}^{1}\left(\bar{Q}_{T}\right)$ the integral

$$
\begin{aligned}
& \int_{t_{1}}^{t_{2}} \int_{\Omega} a\left(u_{e}, \nabla u_{e}\right) \cdot \nabla \phi d x d t \\
& \quad=\int_{t_{1}}^{t_{2}} \int_{\Omega} u_{\varepsilon}^{\alpha} A_{e}^{i} \phi_{x_{i}} d x d t \\
& =\int_{t_{1}}^{t_{2}} \int_{\Omega} u_{\varepsilon}^{\alpha}\left(u_{e}^{-\alpha} a^{i}\left(u_{e}, u \varepsilon^{-\alpha}\left(u \varepsilon^{\alpha} \nabla u \varepsilon\right)\right)\right) \phi_{x_{i}} d x d t
\end{aligned}
$$

tends to the integral $\int_{t_{1}}^{t_{2}} \int_{\Omega} a^{i}\left(u, u_{x}\right) \phi_{x_{i}} d x d t$. On the other hand, in view of (8.1) and (8.15) the integral (8.20) tends to the integral $\int_{t_{1}}^{t_{2}} \int_{\Omega} u^{\alpha} A^{i} \phi_{x_{i}} d x d t$. Hence (8.19) implies (8.18).

The remainder of this section is devoted to the proof of (8.19). Applying Proposition 7.1 with $g(\xi)=\xi-\varepsilon$ we derive from (7.16) that

$$
\left.\int_{\Omega}\left(\frac{1}{2} u_{e}^{2}-\varepsilon u_{\varepsilon}\right) d x\right|_{t_{1}} ^{t_{2}}+\int_{t_{1}}^{t_{2}} \int_{\Omega}\left(a^{i}\left(u_{e}, \nabla u_{e}\right) \frac{\partial u}{\partial x_{i}}-f(u-\varepsilon)\right) d x d t=0 .
$$

Applying Proposition 8.2 with $g(\xi)=\xi$ and using that $u=0$ on $S_{T}$ (in view of (8.1), because $u_{e}=\varepsilon$ on $S_{T}$ ) we derive from (8.11)

$$
\left.\int_{\Omega} \frac{1}{2} u^{2} d x\right|_{t_{1}} ^{t_{2}}+\int_{t_{1}}^{t_{2}} \int_{\Omega}\left(u^{\alpha} A^{i} u_{x_{i}}-f u\right) d x d t=0
$$


Using (8.1) we derive from (8.21) and (8.22) that

$$
\lim _{\varepsilon \rightarrow 0} \int_{i_{1}}^{t_{2}} \int_{\Omega} a^{i}\left(u_{e}, \nabla u_{e}\right) \frac{\partial u_{e}}{\partial x_{i}} d x d t=\int_{t_{1}}^{t_{2}} \int_{\Omega} u^{\alpha} A^{i} u_{x_{i}} d x d t
$$

Let $\bar{u}$ be defined by (8.7). Obviously that the following proposition holds (see also [11]).

Proposition 8.3. We have the convergences

$$
\begin{aligned}
u_{\varepsilon}^{-\alpha} a^{i}\left(u_{\varepsilon}, \nabla \bar{u}\right) \longrightarrow u^{-\alpha} a^{i}(u, \nabla \bar{u}) \quad \text { strongly in } L_{m^{\prime}}\left(Q_{T}\right) \text { as } \varepsilon \rightarrow 0 \\
u_{\varepsilon}^{-\alpha} a^{i}(u, \nabla \bar{u}) \longrightarrow u^{-\alpha} a^{i}\left(u, u_{x}\right) \text { strongly in } L_{m^{\prime}}\left(Q_{T}\right) \text { as } \varepsilon_{1} \rightarrow 0 .
\end{aligned}
$$

Using now condition 2) we have

$$
\begin{aligned}
& \nu_{1} \mathcal{K}_{e, \varepsilon_{1}} \\
& :=\nu_{1} \int_{t_{1}}^{t_{2}} \int_{\Omega} u_{\varepsilon}^{l}\left|\nabla u_{\varepsilon}-\nabla \bar{u}\right|^{2}\left(\left|\nabla u_{e}-b\left(u_{\varepsilon}\right)\right|^{m}+\left|\nabla \bar{u}-b\left(u_{\varepsilon}\right)\right|^{m}\right)^{1-2 / m} d x d t \\
& \leq \int_{i_{1}}^{t_{\Omega}} \int_{\Omega}\left(a^{i}\left(u_{\varepsilon}, \nabla u_{\varepsilon}\right)-a^{i}\left(u_{\varepsilon}, \nabla \bar{u}\right)\right)\left(\frac{\partial u}{\partial x_{i}}-\frac{\partial \bar{u}}{\partial x_{i}}\right) d x d t \\
& =\int_{i_{1}}^{t_{2}} \int_{\Omega}\left(a^{i}\left(u_{\varepsilon}, \nabla u_{\varepsilon}\right) \frac{\partial u_{\varepsilon}}{\partial x_{i}}-A_{\varepsilon}^{i} u_{\varepsilon}^{\alpha} \frac{\partial \bar{u}}{\partial x_{i}}-u_{\varepsilon}^{-\alpha} a^{i}\left(u_{\varepsilon}, \nabla \bar{u}\right)\left(u_{\varepsilon}^{\alpha} \frac{\partial u_{\varepsilon}}{\partial x_{i}}-u_{\varepsilon}^{\alpha} \frac{\partial \bar{u}}{\partial x_{i}}\right)\right) d x d t \\
& =: J_{\varepsilon, \epsilon_{1}} \text {. }
\end{aligned}
$$

Using (8.1), (8.2), (8.15), (8.23), (8.24) and letting $\varepsilon \rightarrow 0$ we obtain

$$
\begin{aligned}
& \lim _{\varepsilon \rightarrow 0} J_{\varepsilon, e_{1}} \\
& =\int_{i_{1}} \int_{\Omega}\left(A^{i}\left(u^{\alpha} u_{x_{i}}-u^{\alpha} \frac{\partial \bar{u}}{\partial x_{i}}\right)-u^{-\alpha} a^{i}(u, \nabla \bar{u})\left(u^{\alpha} u_{x_{i}}-u^{\alpha} \frac{\partial \bar{u}}{\partial x_{i}}\right)\right) d x d t \\
& =: \tilde{J}_{\epsilon_{1}} .
\end{aligned}
$$

Using (8.25) and (8.9) we derive from (8.27)

$$
\lim _{e_{1} \rightarrow 0} \tilde{J}_{e_{1}}=0
$$

From (8.27) and (8.28) it follows that there exist subsequences $\left\{\varepsilon_{k}\right\}$ and $\left\{\varepsilon_{1 k}\right\}$ tending to zero such that $\lim _{k \rightarrow \infty} J_{\varepsilon_{k}, e_{1 k}}=0$. Because $0 \leq \mathcal{H}_{\varepsilon_{k}, \varepsilon_{1 k}} \leq J_{\varepsilon_{k}, \varepsilon_{1 k}}$ we derive from here that

$$
\lim _{k \rightarrow \infty} \mathcal{H}_{e_{k}, e_{1 k}}=0
$$


Rewrite $\mathcal{H}_{\varepsilon_{k}, \varepsilon_{1 k}}$ as

$$
\begin{aligned}
\mathcal{H}_{\varepsilon_{k}, \varepsilon_{1 k}} & \\
= & \int_{t_{1}}^{t_{2}} \int_{\Omega} \frac{\left|u_{\varepsilon_{k}}^{\alpha} \nabla u_{\varepsilon_{k}}-u_{\varepsilon_{k}}^{\alpha} \nabla \bar{u}\right|^{2}}{\left(\left|u_{\varepsilon_{k}}^{\alpha} \nabla u_{\varepsilon_{k}}-u_{\varepsilon_{k}}^{\alpha} b\left(u_{\varepsilon_{k}}\right)\right|^{m}+\left|u_{\varepsilon_{k}}^{\alpha} \nabla \bar{u}-u_{\varepsilon_{k}}^{\alpha} b\left(u_{\varepsilon_{k}}\right)\right|^{m}\right)^{(2-m) / m}} d x d t \\
= & : \int_{i_{1}}^{t_{2}} \int_{\Omega} h_{k}(x, t) d x d t .
\end{aligned}
$$

Is should be recalled that $\bar{u}=\sup \left(u-\varepsilon_{1 k}, 0\right)$ herein. From (8.28) and (8.29) it follows that there exist a subsequence $\{k\}$ and a subset $\widetilde{Q} \subset Q_{t_{1}, t_{2}}=\Omega \times\left[t_{1}, t_{2}\right],|\widetilde{Q}|=\left|Q_{t_{1}, t_{2}}\right|$, such that

$$
\lim _{k \rightarrow \infty} h_{k}(x, t)=0 \quad \text { on } \widetilde{Q} .
$$

Without loss of generality we can count that the derivatives $\frac{\partial u^{a+1}}{\partial x_{i}}$ are finite on $\widetilde{Q}(i=$ $1, \ldots, n)$. Then using (8.1) and (8.3), the definition of $\bar{u}$ (see (8.7)), (8.8) and (8.6), and the continuity of the vector function $b(u)$ we can conclude that

$$
\left|u_{\varepsilon_{k}}^{\alpha} \nabla \bar{u}\right| \text { and }\left|u_{\varepsilon_{k}}^{\alpha} b\left(u_{\varepsilon_{k}}\right)\right| \text { are bounded (non-uniformly) on } \widetilde{Q} \text {. }
$$

On the other hand, in view of the definition of the functions $h_{k}$ we can estimate

$$
h_{k}(x, t) \geq \frac{\left(\left|u_{\varepsilon_{k}}^{\alpha} \nabla u_{e_{k}}\right|-c\right)^{2}}{c\left(\left|u_{\varepsilon_{k}}^{\alpha} \nabla u_{e_{k}}\right|+c\right)^{2-m}} \quad((x, t) \in \widetilde{Q})
$$

with some constant $c$ depending on $(x, t)$. Assume that $\left|u_{\varepsilon_{k}}^{\alpha} \nabla u_{\varepsilon_{k}}\right|$ are unbounded at some point $(x, t) \in \widetilde{Q}$. Then for some subsequence $\{k\}$ we have $\left|u_{\varepsilon_{k}}^{\alpha} \nabla u_{\varepsilon_{k}}\right| \rightarrow \infty$ as $k \rightarrow \infty$ and hence (using that $m \in(1,2)$ ) we derive from $(8.33)$ that

$$
\lim _{k \rightarrow \infty} h_{k}(x, t)=\infty \quad \text { on } \widetilde{Q} \text {. }
$$

But this gives a contradiction with (8.31). Hence

$$
\left|u_{\epsilon_{k}}^{\alpha} \nabla u_{\varepsilon_{k}}\right| \quad \text { are bounded (non-uniformly) on } \widetilde{Q} \text {. }
$$

Then from (8.31), (8.30) and (8.35) it follows that the numerators of $h_{k}$ tend to zero on $\widetilde{Q}$ as $k \rightarrow \infty$, i.e.,

$$
\lim _{k \rightarrow \infty}\left|u_{\varepsilon_{k}}^{\alpha} \frac{\partial u_{\varepsilon_{k}}}{\partial x_{i}}-u_{e_{k}}^{\alpha} \frac{\partial \vec{u}}{\partial x_{i}}\right|=0 \quad \text { on } \tilde{Q} \quad(i=1, \ldots, n) .
$$

Remark now that

$$
\lim _{k \rightarrow \infty}\left|u_{\varepsilon_{k}}^{\alpha} \frac{\partial \bar{u}}{\partial x_{i}}-u^{\alpha} \frac{\partial u}{\partial x_{i}}\right|=0 \quad \text { on } \tilde{Q} \quad(i=1, \ldots, n) .
$$

Really, if $(x, t) \in \tilde{Q}$ and $u(x, t)>0$, then $\frac{\partial \tilde{u}(x, t)}{\partial x_{i}}=u_{x_{i}}(x, t)$ for all sufficiently large $k$ and hence (8.37) follows from (8.1). On the other hand, if $(x, t) \in \widetilde{Q}$ and $u(x, t)=0$, then $\frac{\partial \bar{u}(x, t)}{\partial x_{i}}=0$ for any $k$ and hence (8.37) follows from (8.1) and the definition of $\bar{u}_{x_{i}}$.

Finally, from (8.36) and (8.37) it follows obviously that (8.19) holds. Theorem 6.1 (and hence Theorem 1.1) is proved. 


\section{References}

(1] Bamberger, A.: Etude d'une equation doublement non lineaire. Funct. Anal. 24 (1977), $148-155$.

[2] Chen, Y. Z. and E. DiBenedetto: On the local behavior of solutions of singular parabolic equations. Arch. Rat. Mech. Anal. 103 (1983), 319 - 346.

[3] DiBenedetto, E.: On the local behavior of solutions of degenerate parabolic equations with measurable coefficients. Ann. Sci. Norm. Sup. 13 (1986), $485-535$.

[4] Ivanov, A. V.: Hölder estimates for weak solutions of quasilinear doubly degenerate parabolic equations. Zap. Nauchn. Semin. LOMI 171 (1989), 70 - 105.

[5] Ivanov, A. V.: The class $B_{m, l}$ and Hölder estimates for weak solutions of quasilinear doubly degenerate parabolic equations. Part I. Preprint. St.-Petersburg: Otdel Mat. Inst. Steklov, POMI Preprint No. E-11-91 (1991), pp. 1 - 66.

[6] Ivanov, A. V.: The class $B_{m, l}$ and Hölder estimates for weak solutions of quasilinear doubly degenerate parabolic equations. Part II. Preprint. St.-Petersburg: Otdel Mat. Inst. Steklov, POMI Preprint No. E-12-91 (1991), pp. 1 - 53.

[7] Ivanov, A. V.: The class $B_{m, l}$ and Hölder estimates for generalized solutions of quasilinear parabolic equations admitting double degeneracy. Zap. Nauchn. Semin. LOMI 171 (1989), $70-105$.

[8] Ivanov, A. V.: Hölder estimates for equations of the type of slow or normal diffusion. Zap. Nauchn. Semin. LOMI 215 (1994), $130-136$.

(9] Ivanov, A. V.: Hölder estimates for equations of the type of fast diffusion. Algebra i analiz 6 (1994), $101-142$.

[10] Ivanov, A. V.: The maximum modulus estimates for doubly nonlinear parabolic equations of the type of fast diffusion. Preprint. Bonn: Max-Planck-Institut, Preprint Nr. 94-87 (1994), 1 - 13.

[11] Ivanov, A. V. and P. Z. Mkrtychyan: On the existence of Hölder continuous weak solutions of the first boundary value problem for quasilinear doubly degenerate parabolic equations. Zap. Nauchn. Semin. LOMI 182 (1990), 5 - 28.

[12] Ivanov, A. V., Jäger, W. and P. Z. Mkrtychyan: Existence and uniqueness of regular solution of Cauchy-Dirichlet problem for doubly nonlinear parabolic equations. Zap. Nauchn. Semin. LOMI 213 (1994), 48 - 65.

[13] Kalashnikov, A. S.: Some problems of the qualitative theory of nonlinear parabolic equations. Russain Math. Surveys 42 (1987), 122 - 169.

[14] Ladyzhenskaya, O. A., Solonnikov, V. A. and N. N. Ural'tseva: Linear and Quasilinear Equations of Parabolic Type. Moscow: Nauka 1967.

[15] Lions, J. L.: Quelques Methodes de Resolution de Problemes aux Limites non Lineaires. Paris: Dunod 1969.

[16] Porzio, M. M. and V. Vespri: Hölder estimates for local solutions of some doubly nonlinear parabolic equations. J. Diff. Eq. 103 (1993), $146-178$.

[17] Raviart, P. A.: Sur la resolution de certaines equations parabalique non lineaires. Funct. Anal. 5 (1970), 299 - 328.

[18] Vespri, V.: On the local behavior of solutions of a certain class of doubly nonlinear parabolic equations. Manuscr. Math. 75 (1992), $65-80$. 